This item was submitted to Loughborough's Research Repository by the author.

Items in Figshare are protected by copyright, with all rights reserved, unless otherwise indicated.

\title{
Derivative moments for characteristic polynomials from the CUE
}

PLEASE CITE THE PUBLISHED VERSION

http://dx.doi.org/10.1007/s00220-012-1512-1

PUBLISHER

(c) Springer-Verlag

VERSION

AM (Accepted Manuscript)

LICENCE

CC BY-NC-ND 4.0

REPOSITORY RECORD

Winn, Brian. 2019. "Derivative Moments for Characteristic Polynomials from the CUE". figshare. https://hdl.handle.net/2134/15456. 
This item was submitted to Loughborough's Institutional Repository (https://dspace.lboro.ac.uk/) by the author and is made available under the following Creative Commons Licence conditions.

\section{creative
commons}

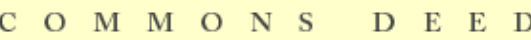

Attribution-NonCommercial-NoDerivs 2.5

You are free:

- to copy, distribute, display, and perform the work

Under the following conditions:

Attribution. You must attribute the work in the manner specified b the author or licensor.

Noncommercial. You may not use this work for commercial purposes.

No Derivative Works. You may not alter, transform, or build upon this work.

- For any reuse or distribution, you must make clear to others the license terms of this work.

- Any of these conditions can be waived if you get permission from the copyright holder.

Your fair use and other rights are in no way affected by the above.

This is a human-readable summary of the Leqal Code (the full license).

\section{Disclaimer 만}

For the full text of this licence, please go to: http://creativecommons.org/licenses/by-nc-nd/2.5/ 


\title{
Derivative moments for characteristic polynomials from the CUE
}

\author{
B. Winn \\ Department of Mathematical Sciences, Loughborough University, \\ Loughborough, LE11 3TU, U.K.
}

$8^{\text {th }}$ December 2011

\begin{abstract}
We calculate joint moments of the characteristic polynomial of a random unitary matrix from the circular unitary ensemble and its derivative in the case that the power in the moments is an odd positive integer. The calculations are carried out for finite matrix size and in the limit as the size of the matrices goes to infinity. The latter asymptotic calculation allows us to prove a long-standing conjecture from random matrix theory.
\end{abstract}

\section{Introduction}

There is a deep, and still only partially-understood, relationship between analytic number theory, and the theory of random matrices. This connection goes back to Montgomery [1] who conjectured that statistical properties of non-trivial zeros of the Riemann zeta function could be predicted by studying the large $N$ asymptotics of correlation functions of eigenvalues of $N \times N$ random unitary matrices. This conjecture is supported by theoretical [1, 2, 3, 4, heuristic [5, 6, 7] and numerical [8, 9, 10] evidence.

The full power of the conjectured relationships between random matrix theory and number theory is found in the study of moments of the Riemann zeta function. Using random matrix theory, mathematicians have been able to make predictions for moments of various kinds, where no conjectures or guesses existed before [11, 12, 13, 14, 15, 16, 17].

A number of review articles have appeared, such as [18, 19, 20, 21], which summarise the main developments that have occurred over the past few years.

The main object of our study will be the $N$-dimensional circular unitary ensemble (CUE) of random matrix theory. This is the probability space consisting of the set $\mathrm{U}(N)$ of $N \times N$ unitary matrices, equipped with normalised Haar measure, $\mu^{\text {Haar }}$.

For a matrix $U \in \mathrm{U}(N)$ we denote the characteristic polynomial by

$$
Z_{U}(\theta):=\prod_{n=1}^{N}\left(1-\mathrm{e}^{\mathrm{i}\left(\theta_{n}-\theta\right)}\right)
$$

where $\mathrm{e}^{\mathrm{i} \theta_{1}}, \ldots, \mathrm{e}^{\mathrm{i} \theta_{N}}$ are the eigenvalues of $U$. 
Define

$$
V_{U}(\theta):=\exp \left(\mathrm{i} N \frac{\theta+\pi}{2}-\mathrm{i} \sum_{n=1}^{N} \frac{\theta_{n}}{2}\right) Z_{U}(\theta) .
$$

Then $V_{U}(\theta)$ is real-valued for $\theta \in[0,2 \pi)$.

In recent years there has been interest in the joint moments of the distribution of $V_{U}$ and its derivative. Define, for $h>-1 / 2$ and $k>h-1 / 2$,

$$
\tilde{F}_{N}(h, k):=\int_{\mathrm{U}(N)}\left|V_{U}(0)\right|^{2 k-2 h}\left|V_{U}^{\prime}(0)\right|^{2 h} \mathrm{~d} \mu^{\text {Haar }},
$$

and the limiting values

$$
\tilde{F}(h, k):=\lim _{N \rightarrow \infty} \frac{1}{N^{k^{2}+2 h}} \tilde{F}_{N}(h, k) .
$$

When $h=0$, the moments 1.3 of $V_{U}$ are precisely the same as the moments of the characteristic polynomial. Keating and Snaith [11] considered $\tilde{F}_{N}(0, k)$, and proved that

$$
\tilde{F}_{N}(0, k)=\prod_{j=1}^{N} \frac{\Gamma(j) \Gamma(j+2 k)}{\Gamma(j+k)^{2}},
$$

and showed that (1.5) extends to the region $\mathfrak{R e}\{k\}>-1 / 2$ of the complex plane.

Let $\mathcal{Z}(t)$ denote Hardy's function:

$$
\mathcal{Z}(t):=\mathrm{e}^{\mathrm{i} \vartheta(t)} \zeta\left(\frac{1}{2}+\mathrm{i} t\right)
$$

where

$$
\vartheta(t):=\mathfrak{I m}\left\{\log \left(\pi^{-\mathrm{i} t / 2} \Gamma\left(\frac{1}{4}+\frac{1}{2} \mathrm{i} t\right)\right)\right\},
$$

and $\zeta(s)$ and $\Gamma(s)$ denote respectively the Riemann zeta function and the Euler gamma function. It follows from the functional equation for $\zeta(s)$ that $\mathcal{Z}(t)$ is real for $t \in \mathbb{R}$, and it is apparent that $\left|\zeta\left(\frac{1}{2}+\mathrm{i} t\right)\right|=|\mathcal{Z}(t)|$, so $\mathcal{Z}(t)$ is to the Riemann zeta function as $V_{U}(\theta)$ is to the characteristic polynomial of a random unitary matrix. A series of conjectures due to Hall [22], Conrey and Ghosh [23], and Hughes [24] has culminated in the following prediction for joint moments of $\mathcal{Z}(t)$ and its derivative:

$$
\frac{1}{T} \int_{0}^{T}|\mathcal{Z}(t)|^{2 k-2 h}\left|\mathcal{Z}^{\prime}(t)\right|^{2 h} \mathrm{~d} t \sim \tilde{F}(h, k) A(k)(\log T)^{k^{2}+2 h}, \quad \text { as } T \rightarrow \infty,
$$

where

$$
A(k):=\prod_{p \text { prime }}\left(1-\frac{1}{p}\right)^{k^{2}} \sum_{\ell=0}^{\infty}\left(\frac{\Gamma(\ell+k)}{\ell ! \Gamma(k)}\right)^{2} p^{-\ell}
$$

Hughes 24] used random matrix theory to calculate $\tilde{F}(h, k)$ for $h=1,2,3$ and Dehaye [25, 26] has derived formulæ for $\tilde{F}(h, k)$ for all $h \in \mathbb{N}$ in terms of sums over partitions (see section 2 below for a precise statement). Using their results, the values $\tilde{F}(1,1)=1 / 12$, $\tilde{F}(1,2)=1 / 720$ and $\tilde{F}(2,2)=1 / 6720$ can be calculated. The corresponding moments (1.8) for Hardy's function have been calculated by Ingham [27] and Conrey [28], and give complete agreement for these values of $k$ and $h$.

Conrey and Ghosh 29] have also proved (assuming the Riemann hypothesis) that

$$
\frac{1}{T} \int_{1}^{T}\left|\mathcal{Z}(t) \mathcal{Z}^{\prime}(t)\right| \mathrm{d} t \sim \frac{\mathrm{e}^{2}-5}{4 \pi}(\log T)^{2} .
$$


This is proved by relating the joint moment to a discrete second moment of the Riemann zeta function at its successive extrema on the critical line, which had been calculated earlier in [30]. The latter result was proved by an integration against the logarithmic derivative of a function with zeros at the locations of maxima of $\left|\zeta\left(\frac{1}{2}+\mathrm{i} t\right)\right|$ and which could be well-approximated by a Dirichlet series. The numerical constants in (1.10) arise as values of residues at poles in the relevant contour integral.

The asymptotic 1.10 naturally leads to the conjecture [24, page 110] that

$$
\tilde{F}\left(\frac{1}{2}, 1\right)=\frac{\mathrm{e}^{2}-5}{4 \pi} .
$$

However, most attention on the problem of calculating moments 1.3 has focussed on integer values of $h$. In this work we will take the first steps beyond integer values of $h$, by studying $\tilde{F}_{N}(h, k)$ for half-integer values of $h$. In particular we will supply a proof of 1.11.

\section{Main results}

In order to put our results into context, we first recall a result of Dehaye [25, 26]. To do this it will be necessary to fix some notations regarding combinatorics of partitions.

We recall that a partition is a finite sequence $\lambda=\left(\lambda_{1}, \ldots, \lambda_{j}\right)$ with $\lambda_{1} \geqslant \lambda_{2} \geqslant \cdots \geqslant \lambda_{j}$. $j$ is the number of parts of $\lambda$, which is also denoted by $\ell(\lambda)$. The sum of the parts of $\lambda$ is denoted by $|\lambda|=\lambda_{1}+\cdots+\lambda_{j}$. For $n \in \mathbb{N}$ we write $\lambda \vdash n$ if $|\lambda|=n$, and $\lambda \vdash_{k} n$ if $|\lambda|=n$ with $\ell(\lambda) \leqslant k$ : a partition of $n$ into not more than $k$ parts.

The generalised Pochhammer symbol $[b]_{\lambda}^{(\sigma)}$ is defined for a partition $\lambda$, a parameter $\sigma>0$ and $b \in \mathbb{R}$ by

$$
[b]_{\lambda}^{(\sigma)}:=\prod_{i=1}^{\ell(\lambda)} \prod_{j=1}^{\lambda_{i}}\left(b+j-1-\frac{i-1}{\sigma}\right) .
$$

We will most often be taking the parameter $\sigma=1$, so that we define the special notation

$$
[b]_{\lambda}:=[b]_{\lambda}^{(1)} .
$$

In terms of the usual (rising) Pochhammer symbol (.)., we have

$$
[b]_{\lambda}=\prod_{i=1}^{\ell(\lambda)}(b-i+1)_{\lambda_{i}} .
$$

A partition can be represented graphically by a Ferrers diagram (see figure 1), in which parts of a partition are represented by a vertical arrangement of boxes aligned at the lefthand side. For each box $\square$ in the Ferrers diagram, the arm-length $a(\square)$ is the number of boxes strictly to the right of $\square$, and the leg-length $g(\square)$ is the number of boxes strictly below $\square$. The hook-length of $\square$ is $a(\square)+g(\square)+1$ : the number of boxes to the right and below, with the box itself counted exactly once. For the partition $\lambda=(4,3,1,1)$, the hook-lengths are indicated in figure 1. The product of all hook-lengths will be called the hook-length of the partition, and denoted $h_{\lambda}$. For example, for $\lambda=(4,3,1,1)$ we find $h_{\lambda}=1680$. 

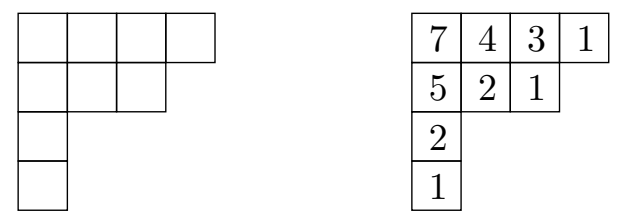

Figure 1: The Ferrers diagram (left) and the hook-lengths (right) for the partition $\lambda=$ $(4,3,1,1)$.

The Ferrers diagram for a partition can be used to define the transpose partition, by reflection of the diagram about the main diagonal. So for $\lambda=(4,3,1,1)$, the transpose partition is $\lambda^{\mathrm{T}}=(4,2,2,1)$. Clearly the length $\ell\left(\lambda^{\mathrm{T}}\right)$ of a transpose partition is equal to the size of the largest part of $\lambda$, and $\left|\lambda^{\mathrm{T}}\right|=|\lambda|$. It is also straightforward to see that

$$
[b]_{\lambda^{\mathrm{T}}}=(-1)^{|\lambda|}[-b]_{\lambda} \quad \text { and } \quad h_{\lambda^{\mathrm{T}}}=h_{\lambda} .
$$

We define two quantities in terms of a sum over partitions. Let $k \in \mathbb{N}$ and $p \in$ $\mathbb{N}_{0}:=\mathbb{N} \cup\{0\}$. Then

$$
\mathcal{C}_{N}(p, k):=(-2)^{p} \sum_{\lambda \vdash_{k} p} \frac{[k]_{\lambda}[-N]_{\lambda}}{[2 k]_{\lambda} h_{\lambda}^{2}},
$$

and

$$
\mathcal{C}(p, k):=2^{p} \sum_{\lambda \vdash_{k} p} \frac{[k]_{\lambda}}{[2 k]_{\lambda} h_{\lambda}^{2}} .
$$

We observe that

$$
\mathcal{C}_{N}(p, k) \sim \mathcal{C}(p, k) N^{p} \quad \text { as } N \rightarrow \infty .
$$

Related quantities appear in the work of Dehaye (see the comment following theorem 2.1 below). In section 7 below we evaluate $\mathcal{C}(p, k)$ explicitly for some small values of $k$.

The main result of Dehaye [25, 26] relevant to our work is the following:

Theorem 2.1. For $h, k \in \mathbb{N}$ with $k>h-\frac{1}{2}$,

$$
\tilde{F}_{N}(h, k)=\frac{(-1)^{h}}{2^{2 h}} \tilde{F}_{N}(0, k) \sum_{p=0}^{2 h} \frac{(2 h) !(-N)^{2 h-p}}{(2 h-p) !} \mathcal{C}_{N}(p, k) .
$$

Moreover, for fixed $h \in \mathbb{N}$, Dehaye has shown that the equation 2.8 extends meromorphically in $k$ to the region $\mathfrak{R e}\{k\}>h-\frac{1}{2}$ of the complex plane.

We have re-written Dehaye's result using our notation. In fact he considers quantities related to $\mathcal{C}_{N}$ and $\mathcal{C}$ (see for example equations (10) and (11) of [26]), which are defined similarly to (2.5) and (2.6), but without the restriction on the number of parts of $\lambda$ in the summation. The presence of the factor $[k]_{\lambda}$ mean that his and our quantities coincide for $p \leqslant 2 k$ (but could be different for $p>2 k$ due to cancellation in that case of factors of the type $0 / 0$ in the numerators and denominators). Since the sum in (2.8) goes only up to $2 h$, this difference is not pertinent in theorem 2.1.

The main result of our work is the following, which gives an explicit formula for $\tilde{F}_{N}(h, k)$ for half-integer $h$ : 
Theorem 2.2. Let $h=(2 m-1) / 2$ for $m \in \mathbb{N}$ and let $k \in \mathbb{N}$ with $k>h-\frac{1}{2}$. Then

$$
\begin{aligned}
\tilde{F}_{N}(h, k)=\frac{2(-1)^{h+1 / 2}}{2^{2 h} \pi} \tilde{F}_{N}(0, k)\left\{\sum_{p=1}^{2 h} \sum_{\ell=1}^{p}\left(\begin{array}{c}
2 h \\
p-\ell
\end{array}\right) \frac{(-1)^{\ell}}{\ell}(-N)^{2 h-p} p ! \mathcal{C}_{N}(p, k)\right. \\
\left.+\sum_{p=2 h+1}^{k N} \frac{(2 h) !(p-2 h-1) !}{N^{p-2 h}} \mathcal{C}_{N}(p, k)\right\} .
\end{aligned}
$$

The paper is structured as follows: In section 3 we write down an integral representation for $\tilde{F}_{N}(h, k)$ involving an integration over a real parameter $\zeta$ and a multi-dimensional integral of size $N$. In section 4 we evaluate the multi-dimensional integral in closed form, and in section 5 we calculate asymptotics of some integrals related to the $\zeta$-integral. We give the proof of theorem 2.2 in section 6 , and we use the theorem to give evaluations of some moments (including (1.11)) in section 7

\section{An integral representation}

\subsection{Notation and properties of Vandermonde determinants}

Let us fix some notation which will be used throughout the remainder of the paper. Let $\mathbf{x}=\left(x_{1}, \ldots, x_{N}\right)$. We will denote in multiple integrals,

$$
\mathrm{d} \mathbf{x}=\mathrm{d} x_{1} \cdots \mathrm{d} x_{N} .
$$

We shall also denote by

$$
\Delta(\mathbf{x}):=\prod_{1 \leqslant j<k \leqslant N}\left(x_{k}-x_{j}\right),
$$

the Vandermonde determinant. It seems prudent at this stage to note a few properties of $\Delta(\cdot)$, that we will make use of later [31].

First of all, note that the Vandermonde determinant is a matrix determinant. We have

$$
\begin{aligned}
\Delta(\mathbf{x}) & =\operatorname{det}\left(x_{i}^{j-1}\right)_{i, j=1, \ldots, N} \\
& =\sum_{\sigma \in S_{N}} \operatorname{sign}(\sigma) x_{1}^{\sigma(1)-1} \cdots x_{N}^{\sigma(N)-1},
\end{aligned}
$$

where $S_{N}$ is the symmetric group on $N$ elements. We see, therefore, that $\Delta(\mathbf{x})$ is a homogeneous polynomial in the variables $x_{1}, \ldots, x_{N}$, of degree $N(N-1) / 2$.

If $\left\{p_{j}(\cdot)\right\}_{j=1, \ldots, N}$ is a set of monic polynomials with the degree of $p_{j}$ being $j-1$, then an alternative expression for $\Delta(\mathbf{x})$ is

$$
\Delta(\mathbf{x})=\operatorname{det}\left(p_{j}\left(x_{i}\right)\right)_{i, j=1, \ldots, N},
$$

which may be proved by applying elementary column operations to the representation (3.3).

In order to justify the convergence of certain integrals, we shall employ the following crude bound. 
Lemma 3.1. For $\mathbf{x} \in \mathbb{R}^{N}$,

$$
|\Delta(\mathbf{x})| \leqslant N !\left(\left(1+x_{1}^{2}\right)^{1 / 2} \cdots\left(1+x_{N}^{2}\right)^{1 / 2}\right)^{N-1} .
$$

Proof. For a permutation $\sigma, \operatorname{sign} \sigma= \pm 1$. So, by (3.4) we can bound

$$
\begin{aligned}
|\Delta(\mathbf{x})| & \leqslant \sum_{\sigma \in S_{N}}\left|x_{1}\right|^{\sigma(1)-1} \cdots\left|x_{N}\right|^{\sigma(N)-1} \\
& \leqslant\left(\sum_{\sigma \in S_{N}} 1\right)\left(1+x_{1}^{2}\right)^{(N-1) / 2} \cdots\left(1+x_{N}^{2}\right)^{(N-1) / 2},
\end{aligned}
$$

and use the fact that $S_{N}$ has order $N$ !.

\subsection{An integral representation for $\tilde{\boldsymbol{F}}_{N}(h, k)$}

To evaluate the averages $\tilde{F}_{N}(h, k)$ we express this quantity as a multi-dimensional integral:

Proposition 3.2. Let $n \in \mathbb{N}_{0}$, and define

$$
K_{n}(\varepsilon, \zeta):=\frac{(-1)^{n}}{\pi} \frac{\partial^{n}}{\partial \varepsilon^{n}}\left(\frac{\varepsilon}{\varepsilon^{2}+\zeta^{2}}\right) .
$$

Then if $2 h \in \mathbb{N}_{0}$ and $k>h-\frac{1}{2}$,

$$
\tilde{F}_{N}(h, k)=\lim _{\varepsilon \downarrow 0} \frac{2^{N^{2}+2 k N-2 h}}{(2 \pi)^{N} N !} \int_{-\infty}^{\infty} \int_{-\infty}^{\infty} \cdots \int_{-\infty}^{\infty} K_{2 h}(\varepsilon, \zeta) \prod_{j=1}^{N} \frac{\mathrm{e}^{\mathrm{i} \zeta x_{j}}}{\left(1+x_{j}^{2}\right)^{N+k}} \Delta(\mathbf{x})^{2} \mathrm{~d} \mathbf{x} \mathrm{d} \zeta .
$$

Let us recapitulate the main steps that we will follow to compute the right-hand side of (3.9). We will first consider the integration over the variables $x_{1}, \ldots, x_{N}$, for fixed $\zeta$, in section 4. In fact we will find that when $k$ is an integer, the integral is a finite series of elementary functions of $\zeta$. To complete the evaluation we will in section 5 calculate the asymptotics of the integral over $\zeta$ of each term in the finite series, as $\varepsilon \downarrow 0$.

In order to prove proposition 3.2 , let us collect a few auxiliary results.

Lemma 3.3. For $x \in \mathbb{R}$ and $\varepsilon>0$, we have

$$
\int_{-\infty}^{\infty} K_{n}(\varepsilon, \zeta) \mathrm{e}^{\mathrm{i} x \zeta} \mathrm{d} \zeta=|x|^{n} \mathrm{e}^{-\varepsilon|x|}
$$

and the integral converges uniformly in $x$ and $\varepsilon \geqslant \varepsilon_{0}>0$.

Proof. By calculating residues, or the Fourier inversion theorem, we know that for $\varepsilon>0$,

$$
\frac{1}{\pi} \int_{-\infty}^{\infty} \frac{\varepsilon}{\varepsilon^{2}+\zeta^{2}} \mathrm{e}^{\mathrm{i} x \zeta} \mathrm{d} \zeta=\mathrm{e}^{-\varepsilon|x|},
$$

uniformly for $\varepsilon \geqslant \varepsilon_{0}>0$. To justify differentiation under the integral, we note that

$$
K_{n}(\varepsilon, \zeta) \ll \frac{1}{\left(\varepsilon^{2}+\zeta^{2}\right)^{(n+1) / 2}}
$$


for $n \geqslant 1$, so that uniform convergence of the resulting integrals is assured.

To compute averages over $\mathrm{U}(N)$, the most useful tool available is Weyl's integration formula. For any function $f(U)$ of a unitary matrix, which depends only on the $N$ eigenvalues $\mathrm{e}^{\mathrm{i} \theta_{1}}, \ldots, \mathrm{e}^{\mathrm{i} \theta_{N}}$

$$
\int_{\mathrm{U}(N)} f(U) \mathrm{d} \mu^{\text {Haar }}=\frac{1}{(2 \pi)^{N} N !} \int_{0}^{2 \pi} \cdots \int_{0}^{2 \pi} f(U) \prod_{1 \leqslant j<k \leqslant N}\left|\mathrm{e}^{\mathrm{i} \theta_{k}}-\mathrm{e}^{\mathrm{i} \theta_{j}}\right|^{2} \mathrm{~d} \theta_{1} \cdots \mathrm{d} \theta_{N} .
$$

By following the substitutions made in [11, we can express $\tilde{F}(h, k)$ as an integral over $\mathbb{R}^{N}$.

Proposition 3.4. It follows from (3.13) that

$$
\tilde{F}_{N}(h, k)=\frac{2^{N^{2}+2 k N-2 h}}{(2 \pi)^{N} N !} \int_{-\infty}^{\infty} \cdots \int_{-\infty}^{\infty} \prod_{j=1}^{N} \frac{1}{\left(1+x_{j}^{2}\right)^{N+k}}\left|x_{1}+\cdots+x_{N}\right|^{2 h} \Delta(\mathbf{x})^{2} \mathrm{~d} \mathbf{x} .
$$

Proof. Differentiating (1.2), we get

$$
V_{U}^{\prime}(\theta)=\frac{\mathrm{i} N}{2} V_{U}(\theta)+\frac{V_{U}(\theta)}{Z_{U}(\theta)} Z_{U}^{\prime}(\theta) .
$$

So,

$$
V_{U}^{\prime}(0)=V_{U}(0)\left(\frac{\mathrm{i} N}{2}+\frac{Z_{U}^{\prime}(0)}{Z_{U}(0)}\right) .
$$

Furthermore,

$$
\begin{aligned}
\frac{Z_{U}^{\prime}(\theta)}{Z_{U}(\theta)} & =\frac{\mathrm{d}}{\mathrm{d} \theta} \log \left(Z_{U}(\theta)\right) \\
& =\sum_{n=1}^{N} \frac{\mathrm{ie}^{\mathrm{i}\left(\theta_{n}-\theta\right)}}{1-\mathrm{e}^{\mathrm{i}\left(\theta_{n}-\theta\right)}},
\end{aligned}
$$

from (1.1). We then get

$$
\begin{aligned}
V_{U}^{\prime}(0) & =\mathrm{i} V_{U}(0)\left(\frac{N}{2}+\sum_{n=1}^{N} \frac{1}{\mathrm{e}^{-\mathrm{i} \theta_{n}}-1}\right) \\
& =-\frac{1}{2} V_{U}(0) \sum_{n=1}^{N} \cot \left(\frac{\theta_{n}}{2}\right)
\end{aligned}
$$

using the fact that

$$
\frac{1}{\mathrm{e}^{-\mathrm{i} \theta_{n}}-1}=\frac{\mathrm{i}}{2} \cot \left(\frac{\theta_{n}}{2}\right)-\frac{1}{2} .
$$

Substituting (3.18) into (1.3), we get

$$
\tilde{F}(h, k)=\frac{1}{2^{2 h}} \int_{\mathrm{U}(N)}\left|V_{U}(0)\right|^{2 k}\left|\sum_{n=1}^{N} \cot \left(\frac{\theta_{n}}{2}\right)\right|^{2 h} \mathrm{~d} \mu^{\text {Haar }} .
$$


Following [11, we write the integral over $\mathrm{U}(N)$ as a multiple-integral using Weyl's identity (3.13), and make the substitutions $x_{j}=\cot \left(\theta_{j} / 2\right)$ therein, leading to (3.14).

Proof of proposition 3.2 . By lemma 3.3 , for any $\varepsilon>0$,

$$
\begin{aligned}
\int_{-\infty}^{\infty} \cdots \int_{-\infty}^{\infty} & \int_{-\infty}^{\infty} K_{2 h}(\varepsilon, \zeta) \prod_{j=1}^{N} \frac{\mathrm{e}^{\mathrm{i} \zeta x_{j}}}{\left(1+x_{j}^{2}\right)^{N+k}} \Delta(\mathbf{x})^{2} \mathrm{~d} \zeta \mathrm{d} \mathbf{x}= \\
& \quad \int_{-\infty}^{\infty} \cdots \int_{-\infty}^{\infty} \prod_{j=1}^{N} \frac{1}{\left(1+x_{j}^{2}\right)^{N+k}}\left|x_{1}+\cdots+x_{N}\right|^{2 h} \mathrm{e}^{-\varepsilon\left|x_{1}+\cdots+x_{N}\right|} \Delta(\mathbf{x})^{2} \mathrm{~d} \mathbf{x} .
\end{aligned}
$$

By lemma 3.1 and equation 3.12 we see that the left-hand side of (3.21) is absolutely integrable, so by the Fubini-Tonelli theorem, we can move the $\zeta$-integral to the outside.

Considering the right-hand side of (3.21), we estimate

$$
\begin{aligned}
\left|x_{1}+\cdots+x_{N}\right|^{2 h} & \leqslant\left(\sqrt{1+x_{1}^{2}}+\cdots+\sqrt{1+x_{N}^{2}}\right)^{2 h} \\
& \leqslant N^{2 h}\left(\left(1+x_{1}^{2}\right)^{1 / 2} \cdots\left(1+x_{N}^{2}\right)^{1 / 2}\right)^{2 h} \\
& =N^{2 h}\left(1+x_{1}^{2}\right)^{h} \cdots\left(1+x_{N}^{2}\right)^{h} .
\end{aligned}
$$

Combining this with lemma 3.1 , we get

$$
\prod_{j=1}^{N} \frac{1}{\left(1+x_{j}^{2}\right)^{N+k}}\left|x_{1}+\cdots+x_{N}\right|^{2 h} \Delta(\mathbf{x})^{2} \leqslant\left(N^{h} N !\right)^{2} \prod_{j=1}^{N} \frac{1}{\left(1+x_{j}^{2}\right)^{k+1-h}},
$$

so that if $k>h-1 / 2$, the right-hand side of (3.21) is uniformly convergent in $\varepsilon$, and we may pass the limit $\varepsilon \downarrow 0$ under the integration. The resulting equality is (3.9).

\section{Evaluation of the multi-dimensional integrals}

The main calculation in this paper is an evaluation of the integral

$$
H(k, \zeta):=\int_{-\infty}^{\infty} \cdots \int_{-\infty}^{\infty} \prod_{j=1}^{N} \frac{\mathrm{e}^{\mathrm{i} \zeta x_{j}}}{\left(1+x_{j}^{2}\right)^{N+k}} \Delta(\mathbf{x})^{2} \mathrm{~d} \mathbf{x},
$$

which appears integrated over $\zeta$ in $(3.9)$. To this end, we will first derive an equivalent representation for $H(k, \zeta)$ which we will be able to evaluate, for integer $k$, in terms of Laguerre polynomials, and multivariable hypergeometric functions.

Proposition 4.1. Let $\zeta>0$ be fixed, and $k \in \mathbb{C}$ with $\mathfrak{R e}\{k\}>-\frac{1}{2}$. Then

$$
H(k, \zeta)=\frac{\pi^{N}}{2^{(N+2 k-1) N}} \prod_{j=0}^{N-1} \frac{1}{\Gamma(k+1+j)^{2}} \mathrm{e}^{-N \zeta} \int_{0}^{\infty} \cdots \int_{0}^{\infty} \prod_{j=1}^{N}\left(y_{j}+2 \zeta\right)^{k} y_{j}^{k} \mathrm{e}^{-y_{j}} \Delta(\mathbf{y})^{2} \mathrm{~d} \mathbf{y} .
$$


Proof. Let

$$
L(k, \zeta):=\int_{0}^{\infty} \cdots \int_{0}^{\infty} \prod_{j=1}^{N}\left(y_{j}+2 \zeta\right)^{k} y_{j}^{k} \mathrm{e}^{-y_{j}} \Delta(\mathbf{y})^{2} \mathrm{~d} \mathbf{y} .
$$

By (3.5) we may write, for fixed $\zeta$,

$$
\begin{aligned}
\Delta(\mathbf{y}) & =\operatorname{det}\left(\left(y_{i}+2 \zeta\right)^{j-1}\right)_{i, j=1, \ldots, N} \\
& =\sum_{\sigma \in S_{N}} \operatorname{sign}(\sigma)\left(y_{1}+2 \zeta\right)^{\sigma(1)-1} \cdots\left(y_{N}+2 \zeta\right)^{\sigma(N)-1} .
\end{aligned}
$$

Using the expression (3.4) for the second of the two Vandermonde factors, we can express $L(k, \zeta)$ as a sum of products of integrals. We get

$$
L(k, \zeta)=\sum_{\sigma, \tau \in S_{N}} \operatorname{sign}(\sigma) \operatorname{sign}(\tau) \prod_{j=1}^{N} \mathcal{I}_{\sigma(j)-1, \tau(j)-1},
$$

where

$$
\mathcal{I}_{\mu, \nu}:=\int_{0}^{\infty}(y+2 \zeta)^{\nu+k} y^{\mu+k} \mathrm{e}^{-y} \mathrm{~d} y .
$$

Let us now consider the integral

$$
\int_{-\infty}^{\infty} \cdots \int_{-\infty}^{\infty} \prod_{j=1}^{N} \frac{\mathrm{e}^{\mathrm{i} \zeta x_{j}+\zeta}}{\left(1+x_{j}^{2}\right)^{N+k}} \Delta(\mathbf{x})^{2} \mathrm{~d} \mathbf{x}
$$

We exploit the homogeneity of $\Delta(\mathbf{x})$ to write

$$
\begin{aligned}
\prod_{j=1}^{N} \frac{1}{\left(1+x_{j}^{2}\right)^{N-1} \Delta(\mathbf{x})^{2}} & =\prod_{1 \leqslant j<m \leqslant N} \frac{\left(x_{m}-x_{j}\right)^{2}}{\left(1+x_{j}^{2}\right)\left(1+x_{m}^{2}\right)} \\
& =\prod_{1 \leqslant j<m \leqslant N} \frac{x_{m}-x_{j}}{\left(1+\mathrm{i} x_{j}\right)\left(1+\mathrm{i} x_{m}\right)} \prod_{1 \leqslant j<m \leqslant N} \frac{x_{m}-x_{j}}{\left(1-\mathrm{i} x_{j}\right)\left(1-\mathrm{i} x_{m}\right)} \\
& =\prod_{1 \leqslant j<m \leqslant N}\left(\frac{1}{1+\mathrm{i} x_{m}}-\frac{1}{1+\mathrm{i} x_{j}}\right) \prod_{1 \leqslant j<m \leqslant N}\left(\frac{1}{1-\mathrm{i} x_{m}}-\frac{1}{1-\mathrm{i} x_{j}}\right) \\
=\sum_{\sigma, \tau \in S_{N}} & \frac{\operatorname{sign}(\sigma) \operatorname{sign}(\tau)}{\left(1+\mathrm{i} x_{1}\right)^{\tau(1)-1}\left(1-\mathrm{i} x_{1}\right)^{\sigma(1)-1} \cdots\left(1+\mathrm{i} x_{N}\right)^{\tau(N)-1}\left(1-\mathrm{i} x_{N}\right)^{\sigma(N)-1}} .
\end{aligned}
$$

Substituting this into 4.7) we get

$$
\int_{-\infty}^{\infty} \cdots \int_{-\infty}^{\infty} \prod_{j=1}^{N} \frac{\mathrm{e}^{\mathrm{i} \zeta x_{j}+\zeta}}{\left(1+x_{j}^{2}\right)^{N+k}} \Delta(\mathbf{x})^{2} \mathrm{~d} \mathbf{x}=\sum_{\sigma, \tau \in S_{N}} \operatorname{sign}(\sigma) \operatorname{sign}(\tau) \prod_{j=1}^{N} \mathcal{J}_{\sigma(j)-1, \tau(j)-1},
$$

where

$$
\mathcal{J}_{\mu, \nu}:=\int_{-\infty}^{\infty} \frac{\mathrm{e}^{\mathrm{i} \zeta x+\zeta}}{(1+\mathrm{i} x)^{1+k+\nu}(1-\mathrm{i} x)^{1+k+\mu}} \mathrm{d} x .
$$

We will now show that $\mathcal{J}_{\mu, \nu}$ is equal to $\mathcal{I}_{\mu, \nu}$, up to a constant independent of $\zeta$. 
We, temporarily, assume that $\mathfrak{R e}\{k\}>0$. Since

$$
\frac{1}{(1-\mathrm{i} x)^{1+k+\mu}}=\frac{1}{\Gamma(1+k+\mu)} \int_{0}^{\infty} \mathrm{e}^{-(1-\mathrm{i} x) u} u^{k+\mu} \mathrm{d} u
$$

we may write

$$
\mathcal{J}_{\mu, \nu}=\frac{1}{\Gamma(1+k+\mu)} \int_{-\infty}^{\infty} \int_{0}^{\infty} \mathrm{e}^{-(1-\mathrm{i} x) u} u^{k+\mu} \frac{\mathrm{e}^{\mathrm{i} \zeta x-\zeta}}{(1+\mathrm{i} x)^{1+k+\nu}} \mathrm{d} u \mathrm{~d} x .
$$

We bound

$$
\left|\frac{1}{(1+\mathrm{i} x)^{1+k+\nu}}\right| \leqslant \frac{\mathrm{e}^{(\pi / 2) \mathfrak{I m}\{k\}}}{\left(1+x^{2}\right)^{(1+\nu+\Re \mathfrak{R}\{k\}) / 2}},
$$

and

$$
\left|\mathrm{e}^{-(1-\mathrm{i} x) u} u^{k+\mu}\right| \leqslant u^{\Re \mathfrak{R}\{k\}+\mu} \mathrm{e}^{-u},
$$

so that the double integral 4.12 is absolutely convergent and we may reverse the order of integration. Therefore, we have

$$
\begin{aligned}
\mathcal{J}_{\mu, \nu} & =\frac{1}{\Gamma(1+k+\mu)} \int_{0}^{\infty} \mathrm{e}^{\zeta-u} u^{k+\mu} \int_{-\infty}^{\infty} \frac{\mathrm{e}^{\mathrm{i}(\zeta+u) x}}{(1+\mathrm{i} x)^{1+k+\nu}} \mathrm{d} x \mathrm{~d} u \\
& =\frac{2 \pi}{\Gamma(1+k+\mu) \Gamma(1+k+\nu)} \int_{0}^{\infty} \mathrm{e}^{\zeta-u} u^{k+\mu}(u+\zeta)^{k+\nu} \mathrm{e}^{-(\zeta+u)} \mathrm{d} u \\
& =\frac{\pi}{2^{2 k+\mu+\nu} \Gamma(1+k+\mu) \Gamma(1+k+\nu)} \int_{0}^{\infty}(y+2 \zeta)^{k+\nu} y^{k+\mu} \mathrm{e}^{-y} \mathrm{~d} y \\
& =\frac{\pi}{2^{2 k+\mu+\nu} \Gamma(1+k+\mu) \Gamma(1+k+\nu)} \mathcal{I}_{\mu, \nu},
\end{aligned}
$$

using Laplace's formula (equation 3.382.6 of [32])

$$
\frac{1}{2 \pi} \int_{-\infty}^{\infty} \frac{\mathrm{e}^{\mathrm{i} p x}}{(1+\mathrm{i} x)^{s}} \mathrm{~d} x=\frac{p^{s-1} \mathrm{e}^{-p}}{\Gamma(s)}, \quad p>0,
$$

to pass from the first to the second line of 4.15.

The relationship (4.15), together with $(4.9)$ and $(4.5)$ proves 4.2 for $\mathfrak{R e}\{k\}>0$. To complete the proof we note that both sides of (4.2) may be continued as analytic functions of $k$ to $\mathfrak{R e}\{k\}>-\frac{1}{2}$.

For integer values of $k$ we are able to give two direct evaluations of the integral in the right hand side of (4.2). The first one uses Laguerre polynomials, and the second uses a hypergeometric function of matrix argument.

\subsection{Evaluation in terms of Laguerre polynomials}

We recall that the classical Laguerre polynomials $L_{n}^{(\alpha)}$ are defined for a parameter $\alpha>-1$ by

$$
L_{n}^{(\alpha)}(t):=\frac{\mathrm{e}^{t}}{t^{\alpha} n !} \frac{\mathrm{d}^{n}}{\mathrm{~d} t^{n}}\left(t^{\alpha+n} \mathrm{e}^{-t}\right) .
$$

An explicit formula for $L_{n}^{(\alpha)}$ is given by

$$
L_{n}^{(\alpha)}(t)=\sum_{j=0}^{n} \frac{\Gamma(n+\alpha+1)}{\Gamma(j+\alpha+1)(n-j) !} \frac{(-t)^{j}}{j !} .
$$


In terms of hypergeometric functions ${ }^{1}$ there is the following expression [33, formula 22.5.54]:

$$
L_{n}^{(\alpha)}(t)=\frac{\Gamma(\alpha+n+1)}{\Gamma(\alpha+1) n !}{ }_{1} F_{1}(-n ; \alpha+1 ; t)
$$

Applying Kummer's transformation [33, formula 13.1.27] to 4.19] leads to an alternative expression,

$$
L_{n}^{(\alpha)}(t)=\mathrm{e}^{t} \sum_{j=0}^{\infty} \frac{\Gamma(\alpha+j+n)}{\Gamma(\alpha+j+1) n !} \frac{(-t)^{j}}{j !} .
$$

We denote by $\mathcal{W}\left(g_{1}, \ldots, g_{n}\right)(x)$ the Wronskian of the $n$ functions $g_{1}, \ldots, g_{n}$, evaluated at $x$ :

$$
\mathcal{W}\left(g_{1}, \ldots, g_{n}\right):=\operatorname{det}\left(\begin{array}{cccc}
g_{1} & g_{2} & \cdots & g_{n} \\
g_{1}^{\prime} & g_{2}^{\prime} & \cdots & g_{n}^{\prime} \\
\vdots & \vdots & \ddots & \vdots \\
g_{1}^{(n-1)} & g_{2}^{(n-1)} & \cdots & g_{n}^{(n-1)}
\end{array}\right)
$$

We have,

Proposition 4.2. For $k \in \mathbb{N}$ and $\zeta \in \mathbb{R}$,

$$
H(k, \zeta)=(-1)^{k(k-1) / 2} \frac{(2 \pi)^{N} N !}{2^{2 k N+N^{2}}} \mathrm{e}^{-N|\zeta|} \mathcal{W}\left(L_{N}^{(k)}, L_{N+1}^{(k)}, \ldots, L_{N+k-1}^{(k)}\right)(-2|\zeta|) .
$$

Proof. The integral on the right-hand side of 4.2 is the averaged moment of the characteristic polynomial of random matrices from the Laguerre unitary ensemble. Such averages were considered by Brézin and Hikami, who showed [34, page 114] that

$$
\int_{0}^{\infty} \cdots \int_{0}^{\infty} \prod_{j=1}^{N}\left(t-y_{j}\right)^{k} y_{j}^{k} \mathrm{e}^{-y_{j}} \Delta(\mathbf{y})^{2} \mathrm{~d} \mathbf{y}=\frac{N ! \prod_{j=0}^{N-1} c_{j}}{\prod_{\ell=0}^{k-1} \ell !} \mathcal{W}\left(p_{N}, \ldots, p_{N+k-1}\right)(t),
$$

where $p_{j}=(-1)^{j} j ! L_{j}^{(k)}$, and

$$
\begin{aligned}
c_{j} & =\int_{0}^{\infty} p_{j}(y)^{2} y^{k} \mathrm{e}^{-y} \mathrm{~d} y \\
& =j !^{2} k !\left(\begin{array}{c}
j+k \\
k
\end{array}\right)=j !(j+k) !
\end{aligned}
$$

(the last line being a classical result of Laguerre polynomials [35]). We have

$$
\begin{aligned}
\mathcal{W}\left(p_{N}, \ldots, p_{N+k-1}\right) & =(-1)^{k N+k(k-1) / 2} \prod_{j=0}^{k-1}(N+j) ! \mathcal{W}\left(L_{N}^{(k)}, \ldots, L_{N+k-1}^{(k)}\right) \\
& =(-1)^{k N+k(k-1) / 2} \prod_{j=N-k}^{N-1}(k+j) ! \mathcal{W}\left(L_{N}^{(k)}, \ldots, L_{N+k-1}^{(k)}\right)
\end{aligned}
$$

\footnotetext{
${ }^{1}$ For the definition of ${ }_{1} F_{1}$, we refer the reader to section 4.2
} 
and

$$
\begin{aligned}
\frac{\prod_{j=0}^{N-1} c_{j}}{\prod_{\ell=0}^{k-1} \ell !} & =\prod_{j=k}^{N-1} j ! \prod_{j=0}^{N-1}(j+k) ! \\
& =\prod_{j=0}^{N-k-1}(j+k) ! \prod_{j=0}^{N-1}(j+k) !
\end{aligned}
$$

Putting 4.25 and 4.26) into 4.23), and setting $t=-2 \zeta$ gives

$$
\begin{aligned}
\int_{0}^{\infty} \cdots \int_{0}^{\infty} \prod_{j=1}^{N}\left(y_{j}+2 \zeta\right)^{k} y_{j}^{k} \mathrm{e}^{-y_{j}} \Delta(\mathbf{y})^{2} \mathrm{~d} \mathbf{y} \\
\quad=(-1)^{k(k-1) / 2} N ! \prod_{j=0}^{N-1}(j+k) !^{2} \mathcal{W}\left(L_{N}^{(k)}, L_{N+1}^{(k)}, \ldots, L_{N+k-1}^{(k)}\right)(-2 \zeta) .
\end{aligned}
$$

Together with proposition 4.1, this proves 4.22 for $\zeta>0$. For general $\zeta \in \mathbb{R}$, we note that the function $H(k, \zeta)$ defined by (4.1) is an even continuous function of $\zeta$, so that we may replace $\zeta$ by $|\zeta|$ wherever it occurs.

For $k=1$, the evaluation 4.22 reduces to

$$
H(1, \zeta)=\frac{\pi^{N} N !}{2^{N^{2}+N}} \mathrm{e}^{-N|\zeta|} L_{N}^{(1)}(-2|\zeta|),
$$

so that the integral (4.1) is proportional to a single Laguerre polynomial. Although we were not able to find a use for $(4.22$ for general $k$, we present in appendix $\mathrm{A}$ an elementary proof of (1.11, based on 4.28, and properties of Laguerre polynomials.

We are also able to write the Wronskian appearing in 4.22 as a Hankel determinant without derivatives, which may be of independent interest.

Proposition 4.3. If $L_{N}^{(k)}$ denotes a Laguerre polynomial, then

$$
\mathcal{W}\left(L_{N}^{(k)}, \ldots, L_{N+k-1}^{(k)}\right)(t)=\operatorname{det}\left(L_{N+k-1-(i+j)}^{(2 k-1)}(t)\right)_{i, j=0, \ldots, k-1} .
$$

Proof. We make repeated use of the identity

$$
\frac{\mathrm{d}}{\mathrm{d} t} L_{n}^{(\alpha)}(t)=-L_{n-1}^{(\alpha+1)}(t),
$$

to get

$$
\begin{aligned}
\mathcal{W}\left(L_{N}^{(k)}, \ldots, L_{N+k-1}^{(k)}\right)(t) & =\operatorname{det}\left(\frac{\mathrm{d}^{j}}{\mathrm{~d} t^{j}} L_{N+i}^{(k)}(t)\right)_{i, j=0, \ldots, k-1} \\
& =\operatorname{det}\left((-1)^{j} L_{N+i-j}^{(k+j)}(t)\right)_{i, j=0, \ldots, k-1} \\
& =(-1)^{\omega} \operatorname{det}\left(L_{N+i-j}^{(k+j)}(t)\right)_{i, j=0, \ldots, k-1},
\end{aligned}
$$

where $\omega=k / 2$ if $k$ is even and $(k-1) / 2$ if $k$ is odd. By application of the identity

$$
L_{n}^{(\alpha-1)}(t)+L_{n-1}^{(\alpha)}(t)=L_{n}^{(\alpha)}(t)
$$


via row operations on the matrix in 4.31, we get

$$
\mathcal{W}\left(L_{N}^{(k)}, \ldots, L_{N+k-1}^{(k)}\right)(t)=(-1)^{\omega} \operatorname{det}\left(L_{N+i-j}^{(2 k-1)}(t)\right)_{i, j=0, \ldots, k-1} .
$$

Finally, switching rows according to $j \mapsto k-1-j$ gives $\omega$ transpositions, leading to 4.29 .

\subsection{Evaluation in terms of hypergeometric functions of matrix argument}

The (single-variable) hypergeometric function ${ }_{p} F_{q}$ is defined formally [36] by the series

$$
{ }_{p} F_{q}\left(a_{1}, \ldots, a_{p} ; b_{1}, \ldots b_{q} ; x\right):=\sum_{j=0}^{\infty} \frac{\left(a_{1}\right)_{j} \cdots\left(a_{p}\right)_{j}}{\left(b_{1}\right)_{j} \cdots\left(b_{q}\right)_{j}} \frac{x^{j}}{j !}
$$

where $(\cdot)$. is the rising Pochhammer symbol. The parameters $a_{1}, \ldots, a_{p}, b_{1}, \ldots, b_{q}$ can be arbitrary complex numbers, however if $b_{i} \in \mathbb{Z} \backslash \mathbb{N}$ then the series 4.34 becomes undefined unless there is a coresponding parameter $a_{i^{\prime}} \in \mathbb{Z}$ with $b_{i}<a_{i^{\prime}} \leqslant 0$, in which case we adopt the convention that the series 4.34 terminates after $a_{i^{\prime}}$ terms.

The hypergeometric functions of a matrix argument provide a multi-variable generalisation of (4.34). They have been studied in [37, 38, 39], and have been found to occur in the context of random matrix theory in the statistics of extreme eigenvalues [40, 41, 42, 43] and moments of characteristic polynomials off the critical line [44, 45], amongst other places. To generalise (4.34), the sum over integers in is replaced by a sum over partitions, the Pochhammer symbols are replaced by the generalised Pochhammer symbols defined in section 2, and the univariate monomials $x^{j}$ are replaced by Jack polynomials (see [46] or [47, chapter 12]).

Let $\sigma>0$ be a parameter, and $X$ be an $N \times N$ matrix with eigenvalues $x_{1}, \ldots, x_{N}$. Then

$$
\begin{aligned}
{ }_{p} F_{q}^{(\sigma)}\left(a_{1}, \ldots a_{p} ; b_{1}, \ldots, b_{q} ; X\right) & :={ }_{p} F_{q}^{(\sigma)}\left(a_{1}, \ldots a_{p} ; b_{1}, \ldots, b_{q} ; x_{1}, \ldots, x_{N}\right) \\
& :=\sum_{\lambda} \frac{\left[a_{1}\right]_{\lambda}^{(\sigma)} \cdots\left[a_{p}\right]_{\lambda}^{(\sigma)}}{\left[b_{1}\right]_{\lambda}^{(\sigma)} \cdots\left[b_{q}\right]_{\lambda}^{(\sigma)}} \frac{C_{\lambda}^{(\sigma)}\left(x_{1}, \ldots, x_{N}\right)}{|\lambda| !}
\end{aligned}
$$

where the Jack polynomials $C_{\lambda}^{(\sigma)}$ are [46] homogeneous symmetric polynomial eigenfunctions of the partial differential operator

$$
\mathrm{D}(\sigma):=\frac{\sigma}{2} \sum_{i=1}^{N} x_{i}^{2} \frac{\partial^{2}}{\partial x_{i}^{2}}+\sum_{i \neq j} \frac{x_{i}^{2}}{x_{i}-x_{j}} \frac{\partial}{\partial x_{i}},
$$

normalised so that

$$
C_{\lambda}^{(\sigma)}(1, \ldots, 1)=\frac{\sigma^{2|\lambda|}|\lambda| !}{c_{\lambda}(\sigma) c_{\lambda}^{\prime}(\sigma)}\left[\frac{N}{\sigma}\right]_{\lambda}^{(\sigma)}
$$

(this is different to the normalisation used in [46]) where $c_{\lambda}(\sigma)$ and $c_{\lambda}^{\prime}(\sigma)$ generalise the hook lengths defined in section 2 , their definitions are

$$
c_{\lambda}(\sigma):=\prod_{\square \in \lambda}(g(\square)+1+\sigma a(\square))
$$


and

$$
c_{\lambda}^{\prime}(\sigma):=\prod_{\square \in \lambda}(g(\square)+\sigma(1+a(\square))) .
$$

For the case $\sigma=1$ the polynomials $C_{\lambda}^{(1)}$ are proportional to Schur polynomials. In that case, the denominator in 4.38 is $c_{\lambda}(1) c_{\lambda}^{\prime}(1)=h_{\lambda}^{2}$.

Jack polynomials have the property [46] that the number of parts of $\lambda$ is restricted by the number of variables; we have

$$
C_{\lambda}^{(\sigma)}\left(x_{1}, \ldots, x_{N}\right)=0 \quad \text { if } \ell(\lambda)>N .
$$

The convergence of the series in (4.34) and 4.36) depends in general on the parameters $a_{1}, \ldots, a_{p}, b_{1}, \ldots, b_{q}$. If $p-q \leqslant 1$ then the radii of convergence in (4.34) and (4.36) are at least 1 . If $a_{i} \in \mathbb{Z} \backslash \mathbb{N}$ for any $i$ then the series terminate, and are thus defined for all values of the arguments (in which case the resulting functions are polynomials). For the single-variable case this is straightforward from the definition. To see why it is also the case for hypergeometric functions of a matrix argument, observe that by $[2.1],[a]_{\lambda}^{(\sigma)}=0$ if $\lambda_{1}>-a$ for $a$ a negative integer. This restricts the horizontal number of boxes in the partition $\lambda$, and property (4.41) restricts the vertical number of partitions. Together these implies that the series 4.36) contains only a finite number of non-zero terms.

Hypergeometric functions of matrix argument enjoy a reflection property [48, page 812] that we will make use of. Let $a \in \mathbb{N}$ and $b>a$. Then,

$$
\begin{aligned}
& { }_{1} F_{1}^{(\sigma)}\left(-a ;-b ; x_{1}, \ldots, x_{n}\right)= \\
& \left(x_{1} \cdots x_{n}\right)^{a} \prod_{j=1}^{n} \frac{\Gamma\left(b-a+\frac{j-1}{\sigma}+1\right)}{\Gamma\left(b+\frac{j-1}{\sigma}+1\right)}{ }_{2} F_{0}^{(\sigma)}\left(-a, 1+b-a+\frac{n-1}{\sigma} ; ; \frac{-1}{x_{1}}, \ldots, \frac{-1}{x_{n}}\right) .
\end{aligned}
$$

This may be viewed as a generalisation of the single-variable identity:

$$
{ }_{1} F_{1}(-a ;-b ; x)=x^{a} \frac{\Gamma(b-a+1)}{\Gamma(b+1)}{ }_{2} F_{0}\left(-a, 1+b-a ; ; \frac{-1}{x}\right) .
$$

In both (4.42) and 4.43) we emphasise that since the parameter $a$ is a negative integer, the hypergeometric series are actually finite, and the left-hand side series terminate before the denominators in the summands in 4.34 ) and 4.36 become zero in the case that we additionally have $b \in \mathbb{N}$.

The main result of this section is the following:

Proposition 4.4. For $k \in \mathbb{N}$ and $\zeta \in \mathbb{R}$,

$$
H(k, \zeta)=\frac{\pi^{N} N !}{2^{(N+2 k-1) N}} \tilde{F}_{N}(0, k) \mathrm{e}^{-N|\zeta|}{ }_{1} F_{1}^{(1)}(-k ;-2 k ; 2|\zeta|, \ldots, 2|\zeta|) .
$$

The proof of proposition 4.4 is based on the following evaluation of the integral $L(k, \zeta)$ :

Proposition 4.5. Let $L$ denote the integral in (4.3), and let $k \in \mathbb{N}$ and $\zeta>0$. Then

$$
L(k, \zeta)=\left(\prod_{j=0}^{N-1} \Gamma(j+2) \Gamma(k+1+j)\right)(2 \zeta)^{k N}{ }_{2} F_{0}^{(1)}\left(-k ; N+k ; ; \frac{-1}{2 \zeta}, \ldots, \frac{-1}{2 \zeta}\right) .
$$


Proof. Forrester and Keating [45, equation (3.2)] have proved ${ }^{2}$ the following integral, valid for $\mathfrak{R e}\{a\}>-1, \mathfrak{R e}\{b\}>-1$ and either $|t|>1$ or $2 \mu \in \mathbb{N}$ :

$$
\begin{aligned}
& \frac{1}{S_{N}(a+1, b+1, \gamma)} \int_{0}^{1} \cdots \int_{0}^{1} \prod_{j=1}^{N} x_{j}^{a}\left(1-x_{j}\right)^{b}\left(t-x_{j}\right)^{2 \mu}|\Delta(\mathbf{x})|^{2 \gamma} \mathrm{d} \mathbf{x} \\
& \quad=t_{2}^{2 \mu N} F_{1}^{(1 / \gamma)}\left(-2 \mu, \gamma(N-1)+a+1 ; 2 \gamma(N-1)+a+b+2 ; \frac{1}{t}, \ldots, \frac{1}{t}\right),
\end{aligned}
$$

where

$$
S_{N}(\alpha, \beta, \gamma):=\prod_{j=0}^{N-1} \frac{\Gamma(\alpha+j \gamma) \Gamma(\beta+j \gamma) \Gamma(1+(j+1) \gamma)}{\Gamma(\alpha+\beta+(N+j-1) \gamma) \Gamma(1+\gamma)} .
$$

We let $2 \mu=k \in \mathbb{N}$ and $a=k, b=B, \gamma=1, t=-2 \zeta / B$, and make the changes of variables $x_{j}=y_{j} / B$. The integral on the left-hand side of (4.46) becomes

$$
\frac{(-1)^{k N}}{B^{N^{2}+2 k N}} \int_{0}^{B} \cdots \int_{0}^{B} \prod_{j=1}^{N} y_{j}^{k}\left(1-\frac{y_{j}}{B}\right)^{B}\left(y_{j}+2 \zeta\right)^{k} \Delta(\mathbf{y})^{2} \mathrm{~d} \mathbf{y} .
$$

Since $\left(1-y_{j} / B\right)^{B} \rightarrow \mathrm{e}^{-y_{j}}$ as $B \rightarrow \infty$, and

$$
\left(1-\frac{y_{j}}{B}\right)^{B} \mathbb{1}_{[0, B]}(y) \leqslant \mathrm{e}^{-y_{j}},
$$

for $y_{j} \geqslant 0$, we get, by the dominated convergence theorem,

$$
\begin{aligned}
& L(k, \zeta)=\lim _{B \rightarrow \infty}(2 \zeta)^{k N} \\
& \quad \times B^{N^{2}+k N} S_{N}(k+1, B+1,1){ }_{2} F_{1}^{(1)}\left(-k, N+k ; 2 N+k+B ; \frac{-B}{2 \zeta}, \ldots, \frac{-B}{2 \zeta}\right),
\end{aligned}
$$

where the hypergeometric function of a matrix argument is a multivariate polynomial since $k \in \mathbb{N}$. By Stirling's formula and 4.47) it follows that

$$
\lim _{B \rightarrow \infty} B^{N^{2}+k N} S_{N}(k+1, B+1,1)=\prod_{j=0}^{N-1} \Gamma(j+2) \Gamma(k+j+1) .
$$

For a partition $\lambda$, it is clear that $[2 N+k+B]_{\lambda} \sim B^{|\lambda|}$ as $B \rightarrow \infty$, and the fact that $C_{\lambda}^{(1)}$ is homogeneous of degree $|\lambda|$ suffices to conclude from (4.36) that

$$
\lim _{B \rightarrow \infty}{ }_{2} F_{1}^{(1)}\left(-k, N+k ; 2 N+k+B ; \frac{-B}{2 \zeta}, \ldots, \frac{-B}{2 \zeta}\right)={ }_{2} F_{0}^{(1)}\left(-k, N+k ; ; \frac{-1}{2 \zeta}, \ldots, \frac{-1}{2 \zeta}\right) .
$$

This completes the proof.

Similar integrals have been evaluated in [48] by a different method.

Proof of proposition 4.4. Combining the result of the proposition 4.5 with the reflection formula (4.42) and equation (4.2) leads to (4.44), for $\zeta>0$. To pass to the case $\zeta \in \mathbb{R}$ we use the same argument as at the end of the proof of proposition 4.2 .

\footnotetext{
${ }^{2}$ There is a typo in equation (3.2) of [45] in the second parameter of the hypergeometric function. We have given here a corrected formula.
} 


\section{$5 \quad$ Some integrals involving $K_{n}(\varepsilon, \zeta)$}

In this section we will consider for integer values of $n$ and $p$, integrals of the form

$$
\int_{0}^{\infty} K_{n}(\varepsilon, \zeta) \mathrm{e}^{-N \zeta} \zeta^{p} \mathrm{~d} \zeta
$$

in the asymptotic régime $\varepsilon \downarrow 0$.

The function $K_{n}$ is defined in $(3.8)$ as a partial derivative with respect to $\varepsilon$ of a function of $\varepsilon$ and $\zeta$. It will be convenient to obtain alternative formulæ for $K_{n}$ with derivatives with respect to $\zeta$.

Lemma 5.1. Let $K_{n}(\varepsilon, \zeta)$ be defined by (3.8). If $n=2 m$ is even then

$$
K_{n}(\varepsilon, \zeta)=\frac{(-1)^{m}}{\pi} \frac{\partial^{n}}{\partial \zeta^{n}}\left(\frac{\varepsilon}{\varepsilon^{2}+\zeta^{2}}\right),
$$

whereas if $n=2 m-1$ is odd then

$$
K_{n}(\varepsilon, \zeta)=\frac{(-1)^{m+1}}{\pi} \frac{\partial^{n}}{\partial \zeta^{n}}\left(\frac{\zeta}{\varepsilon^{2}+\zeta^{2}}\right) .
$$

Proof. We begin by observing that

$$
\begin{aligned}
K_{n}(\varepsilon, \zeta) & =\frac{(-1)^{n}}{\pi} \frac{\partial^{n}}{\partial \varepsilon^{n}}\left(\frac{\varepsilon}{\varepsilon^{2}+\zeta^{2}}\right) \\
& =\frac{(-1)^{n}}{2 \pi} \frac{\partial^{n}}{\partial \varepsilon^{n}}\left(\frac{1}{\varepsilon+\mathrm{i} \zeta}+\frac{1}{\varepsilon-\mathrm{i} \zeta}\right) \\
& =\frac{n !}{2 \pi}\left(\frac{1}{\mathrm{i}^{n+1}(\zeta-\mathrm{i} \varepsilon)^{n+1}}+\frac{1}{(-\mathrm{i})^{n+1}(\zeta+\mathrm{i} \varepsilon)^{n+1}}\right) .
\end{aligned}
$$

If $n=2 m-1$ is odd, then

$$
\begin{aligned}
K_{n}(\varepsilon, \zeta) & =\frac{n !}{2 \pi \mathrm{i}^{2 m}}\left(\frac{1}{(\zeta-\mathrm{i} \varepsilon)^{n+1}}+\frac{1}{(\zeta+\mathrm{i} \varepsilon)^{n+1}}\right) \\
& =\frac{(-1)^{n+m}}{2 \pi} \frac{\partial^{n}}{\partial \zeta^{n}}\left(\frac{1}{\zeta-\mathrm{i} \varepsilon}+\frac{1}{\zeta+\mathrm{i} \varepsilon}\right) \\
& =\frac{(-1)^{m+1}}{\pi} \frac{\partial^{n}}{\partial \zeta^{n}}\left(\frac{\zeta}{\varepsilon^{2}+\zeta^{2}}\right) .
\end{aligned}
$$

On the other hand, if $n=2 m$ is even, then from (5.4) we get

$$
\begin{aligned}
K_{n}(\varepsilon, \zeta) & =\frac{(-1)^{n+m}}{2 \pi \mathrm{i}} \frac{\partial^{n}}{\partial \zeta^{n}}\left(\frac{1}{\zeta-\mathrm{i} \varepsilon}-\frac{1}{\zeta+\mathrm{i} \varepsilon}\right) \\
& =\frac{(-1)^{m}}{\pi} \frac{\partial^{n}}{\partial \zeta^{n}}\left(\frac{\varepsilon}{\varepsilon^{2}+\zeta^{2}}\right) .
\end{aligned}
$$


Lemma 5.2. Let $L_{n}^{(\alpha)}$ denote the Laguerre polynomials, defined by 4.17). If $p \geqslant n$ then

$$
\frac{\partial^{n}}{\partial \zeta^{n}}\left(\zeta^{p} \mathrm{e}^{-N \zeta}\right)=n ! \zeta^{p-n} \mathrm{e}^{-N \zeta} L_{n}^{(p-n)}(N \zeta)
$$

For $p \leqslant n$, we have

$$
\frac{\partial^{n}}{\partial \zeta^{n}}\left(\zeta^{p} \mathrm{e}^{-N \zeta}\right)=p !(-N)^{n-p} \mathrm{e}^{-N \zeta} L_{p}^{(n-p)}(N \zeta)
$$

Proof. The case $p \geqslant n$ is slightly the simpler and we consider it first. We have

$$
\begin{aligned}
\frac{\partial^{n}}{\partial \zeta^{n}}\left(\zeta^{p} \mathrm{e}^{-N \zeta}\right) & =N^{-p} \frac{\partial^{n}}{\partial \zeta^{n}}\left((N \zeta)^{p} \mathrm{e}^{-N \zeta}\right) \\
& =\left.N^{n-p} \frac{\partial^{n}}{\partial \xi^{n}}\left(\xi^{p} \mathrm{e}^{-\xi}\right)\right|_{\xi=N \zeta} \\
& =\left.N^{n-p} n ! \xi^{p-n} \mathrm{e}^{-\xi} L_{n}^{(p-n)}(\xi)\right|_{\xi=N \zeta} \\
& =n ! \zeta^{p-n} \mathrm{e}^{-N \zeta} L_{n}^{(p-n)}(N \zeta) .
\end{aligned}
$$

If $p \leqslant n$, we still have

$$
\frac{\partial^{n}}{\partial \zeta^{n}}\left(\zeta^{p} \mathrm{e}^{-N \zeta}\right)=\left.N^{n-p} \frac{\partial^{n}}{\partial \zeta^{n}}\left(\xi^{p} \mathrm{e}^{-\xi}\right)\right|_{\xi=N \zeta},
$$

but now we write

$$
\begin{aligned}
\frac{\partial^{n}}{\partial \zeta^{n}}\left(\zeta^{p} \mathrm{e}^{-N \zeta}\right) & =\left.N^{n-p} \frac{\partial^{n-p}}{\partial \xi^{n-p}}\left(\frac{\partial^{p}}{\partial \xi^{p}}\left(\xi^{p} \mathrm{e}^{-\xi}\right)\right)\right|_{\xi=N \zeta} \\
& =\left.N^{n-p} p ! \frac{\partial^{n-p}}{\partial \xi^{n-p}}\left(\mathrm{e}^{-\xi} L_{p}^{(0)}(\xi)\right)\right|_{\xi=N \zeta}
\end{aligned}
$$

At this point we use 4.20 to write, for $\alpha \in \mathbb{N}_{0}$,

$$
L_{p}^{(\alpha)}(\xi)=\mathrm{e}^{\xi} \sum_{\ell=0}^{\infty} \frac{(\alpha+p+\ell-1) !}{(\alpha+\ell) ! p !} \frac{(-\xi)^{\ell}}{\ell !}
$$

where the power series converges for all values of $\xi$. Thereby we get an expression for $\mathrm{e}^{-\xi} L_{p}^{(0)}(\xi)$ which we may legitimately differentiate term-by-term to get

$$
\begin{aligned}
\frac{\partial^{n-p}}{\partial \xi^{n-p}}\left(\mathrm{e}^{-\xi} L_{p}^{(0)}(\xi)\right) & =\sum_{\ell=n-p}^{\infty} \frac{(p+\ell-1) !}{\ell ! p !} \frac{(-1)^{\ell}}{\ell !} \frac{\ell !}{(\ell-n+p) !} \xi^{\ell-n+p} \\
& =(-1)^{n-p} \sum_{m=0}^{\infty} \frac{(m+n-1) !}{(m+n-p) ! p !} \frac{(-\xi)^{m}}{m !}, \quad \text { via } \ell=m+n-p \\
& =(-1)^{n-p} \mathrm{e}^{-\xi} L_{p}^{(n-p)}(\xi),
\end{aligned}
$$

using (5.12) once more. Substitution of 5.13) into 5.11) completes the proof.

For the case $p>n$ in (5.1), the asymptotic evaluations are given by the following proposition. 
Proposition 5.3. Let $p \geqslant n+1$. Then, as $\varepsilon \downarrow 0$, we have

$$
\int_{0}^{\infty} K_{n}(\varepsilon, \zeta) \mathrm{e}^{-N \zeta} \zeta^{p} \mathrm{~d} \zeta= \begin{cases}\frac{n !(p-n-1) !}{\pi N^{p-n}}(-1)^{(n+1) / 2}+\mathrm{o}(1), & n \text { odd }, \\ \mathrm{o}(1), & n \text { even } .\end{cases}
$$

Proof. We use the representations of $K_{n}(\varepsilon, \zeta)$ derived in lemma 5.1, and then integrate by parts, and insert the derivative formulæ from lemma 5.2 .

If $n=2 m-1$ is odd then

$$
\begin{aligned}
\int_{0}^{\infty} K_{n}(\varepsilon, \zeta) \mathrm{e}^{-N \zeta} \zeta^{p} \mathrm{~d} \zeta & =\frac{(-1)^{m+1}}{\pi} \int_{0}^{\infty} \frac{\partial^{n}}{\partial \zeta^{n}}\left(\frac{\zeta}{\varepsilon^{2}+\zeta^{2}}\right) \mathrm{e}^{-N \zeta} \zeta^{p} \mathrm{~d} \zeta \\
& =\frac{(-1)^{n+m+1}}{\pi} \int_{0}^{\infty} \frac{\zeta}{\varepsilon^{2}+\zeta^{2}} \frac{\partial^{n}}{\partial \zeta^{n}}\left(\mathrm{e}^{-N \zeta} \zeta^{p}\right) \mathrm{d} \zeta \\
& =\frac{(-1)^{m}}{\pi} n ! \int_{0}^{\infty} \frac{\zeta^{p-n+1}}{\varepsilon^{2}+\zeta^{2}} \mathrm{e}^{-N \zeta} L_{n}^{(p-n)}(N \zeta) \mathrm{d} \zeta
\end{aligned}
$$

Since $p-n+1 \geqslant 2$ and since $\left|\zeta^{2} /\left(\varepsilon^{2}+\zeta^{2}\right)\right| \leqslant 1$ the integral in (5.15) is uniformly convergent in $\varepsilon$, so we can pass the limit under the integral and get

$$
\lim _{\varepsilon \downarrow 0} \int_{0}^{\infty} K_{n}(\varepsilon, \zeta) \mathrm{e}^{-N \zeta} \zeta^{p} \mathrm{~d} \zeta=\frac{(-1)^{m}}{\pi} n ! \int_{0}^{\infty} \zeta^{p-n-1} \mathrm{e}^{-N \zeta} L_{n}^{(p-n)}(N \zeta) \mathrm{d} \zeta .
$$

At this point, we insert the expansion 4.18 for the Laguerre polynomial, to find

$$
\begin{aligned}
\lim _{\varepsilon \downarrow 0} \int_{0}^{\infty} K_{n}(\varepsilon, \zeta) \mathrm{e}^{-N \zeta} \zeta^{p} \mathrm{~d} \zeta & =\frac{(-1)^{m}}{\pi} n ! \sum_{\ell=0}^{n}\left(\begin{array}{c}
p \\
n-\ell
\end{array}\right) \frac{(-1)^{\ell}}{\ell !} N^{\ell} \int_{0}^{\infty} \zeta^{p-n+\ell-1} \mathrm{e}^{-N \zeta} \mathrm{d} \zeta \\
& =\frac{(-1)^{m}}{\pi} \frac{n !(p-n-1) !}{N^{p-n}} \sum_{\ell=0}^{n}(-1)^{\ell}\left(\begin{array}{c}
p \\
n-\ell
\end{array}\right)\left(\begin{array}{c}
p-n+\ell-1 \\
\ell
\end{array}\right) \\
& =\frac{(-1)^{m}}{\pi} \frac{n !(p-n-1) !}{N^{p-n}},
\end{aligned}
$$

using that the sum in (5.17) evaluates to 1 , a fact which is proved in appendix B.1.

In the case that $n=2 m$ is even we again use lemma 5.1 and lemma 5.2 to find that

$$
\begin{aligned}
\int_{0}^{\infty} K_{n}(\varepsilon, \zeta) \mathrm{e}^{-N \zeta} \zeta^{p} \mathrm{~d} \zeta & =\frac{(-1)^{m+n}}{\pi} \int_{0}^{\infty} \frac{\varepsilon}{\varepsilon^{2}+\zeta^{2}} \frac{\partial^{n}}{\partial \zeta^{n}}\left(\mathrm{e}^{-N \zeta} \zeta^{p}\right) \mathrm{d} \zeta \\
& =\frac{(-1)^{m+n}}{\pi} n ! \int_{0}^{\infty} \frac{\varepsilon}{\varepsilon^{2}+\zeta^{2}} \zeta^{p-n} \mathrm{e}^{-N \zeta} L_{n}^{(p-n)}(N \zeta) \mathrm{d} \zeta \\
& =\mathrm{o}(1),
\end{aligned}
$$

as $\varepsilon \downarrow 0$, using lemma 5.4 below.

In the proof of propositon 5.3 we used the following standard result (quoted without proof):

Lemma 5.4. If $f$ is a bounded function with $f(x) \rightarrow f_{0}$ as $x \downarrow 0$, then

$$
\int_{0}^{\infty} \frac{\varepsilon}{\varepsilon^{2}+x^{2}} f(x) \mathrm{d} x=\frac{\pi f_{0}}{2}+\mathrm{o}(1)
$$

as $\varepsilon \downarrow 0$. 
Proposition 5.5. If $p \leqslant n$ and $n=2 m$ is even, then

$$
\int_{0}^{\infty} K_{n}(\varepsilon, \zeta) \mathrm{e}^{-N \zeta} \zeta^{p} \mathrm{~d} \zeta=\frac{(-1)^{n / 2}}{2} \frac{n !}{(n-p) !}(-N)^{n-p}+\mathrm{o}(1)
$$

as $\varepsilon \downarrow 0$.

Proof. Applying lemma 5.1 and lemma 5.2 we get

$$
\begin{aligned}
\int_{0}^{\infty} K_{n}(\varepsilon, \zeta) \mathrm{e}^{-N \zeta} \zeta^{p} \mathrm{~d} \zeta & =\frac{(-1)^{m+n}}{\pi} \int_{0}^{\infty} \frac{\varepsilon}{\varepsilon^{2}+\zeta^{2}} \frac{\partial^{n}}{\partial \zeta^{n}}\left(\mathrm{e}^{-N \zeta} \zeta^{p}\right) \mathrm{d} \zeta \\
& =\frac{(-1)^{m+n}}{\pi} p !(-N)^{n-p} \int_{0}^{\infty} \frac{\varepsilon}{\varepsilon^{2}+\zeta^{2}} \mathrm{e}^{-N \zeta} L_{p}^{(n-p)}(N \zeta) \mathrm{d} \zeta .
\end{aligned}
$$

Applying lemma 5.4, we find in the limit $\varepsilon \downarrow 0$,

$$
\begin{aligned}
\int_{0}^{\infty} K_{n}(\varepsilon, \zeta) \mathrm{e}^{-N \zeta} \zeta^{p} \mathrm{~d} \zeta & =\frac{(-1)^{m+n}}{\pi} p !(-N)^{n-p} \frac{\pi}{2} L_{n}^{(n-p)}(0)+\mathrm{o}(1) \\
& =\frac{(-1)^{m+n}}{2} \frac{n !}{(n-p) !}(-N)^{n-p}+\mathrm{o}(1),
\end{aligned}
$$

using the explicit representation 4.18 for the Laguerre polynomial.

For odd $n \geqslant p$, we have the slightly more subtle result:

Proposition 5.6. Let $f(\zeta):=\sum_{p=0}^{P} f_{p} \zeta^{p}$, with $P \leqslant n$, where $n$ is now odd. Then provided that

$$
\sum_{p=0}^{P}\left(\begin{array}{c}
n \\
p
\end{array}\right) \frac{f_{p} p !}{(-N)^{p}}=0
$$

then

$$
\lim _{\varepsilon \downarrow 0} \int_{0}^{\infty} K_{n}(\varepsilon, \zeta) \mathrm{e}^{-N \zeta} f(\zeta) \mathrm{d} \zeta=\frac{(-1)^{(n+1) / 2}}{\pi} \sum_{p=1}^{P} \sum_{\ell=1}^{p} p !\left(\begin{array}{c}
n \\
p-\ell
\end{array}\right) \frac{(-1)^{\ell}(-N)^{n-p}}{\ell} f_{p} .
$$

If condition (5.24) does not hold, then the limit in (5.25) diverges.

Proof. Following the arguments above, we find for $n=2 m-1$ and $p \leqslant n$,

$$
\int_{0}^{\infty} K_{n}(\varepsilon, \zeta) \mathrm{e}^{-N \zeta} \zeta^{p} \mathrm{~d} \zeta=\frac{(-1)^{m}}{\pi} p !(-N)^{n-p} \int_{0}^{\infty} \frac{\zeta}{\varepsilon^{2}+\zeta^{2}} \mathrm{e}^{-N \zeta} L_{p}^{(n-p)}(N \zeta) \mathrm{d} \zeta .
$$

Since

$$
L_{p}^{(n-p)}(N \zeta)=\left(\begin{array}{c}
n \\
p
\end{array}\right)+\sum_{\ell=1}^{p}\left(\begin{array}{c}
n \\
p-\ell
\end{array}\right) \frac{(-N)^{\ell}}{\ell !} \zeta^{\ell}
$$

we have that

$$
\begin{aligned}
& \int_{0}^{\infty} \frac{\zeta \mathrm{e}^{-N \zeta}}{\varepsilon^{2}+\zeta^{2}} L_{p}^{(n-p)}(N \zeta) \mathrm{d} \zeta=\left(\begin{array}{c}
n \\
p
\end{array}\right) \int_{0}^{\infty} \frac{\zeta \mathrm{e}^{-N \zeta}}{\varepsilon^{2}+\zeta^{2}} \mathrm{~d} \zeta \\
&+\sum_{\ell=1}^{p}\left(\begin{array}{c}
n \\
p-\ell
\end{array}\right) \frac{(-N)^{\ell}}{\ell !} \int_{0}^{\infty} \zeta^{\ell-1} \mathrm{e}^{-N \zeta} \mathrm{d} \zeta+\mathrm{o}(1),
\end{aligned}
$$


passing the limit $\varepsilon \downarrow 0$ in a similar way as in the proof of the first part of proposition 5.3 . Evaluating the integral on the right hand side of (5.28) we get

$$
\begin{aligned}
\int_{0}^{\infty} K_{n}(\varepsilon, \zeta) \mathrm{e}^{-N \zeta} \zeta^{p} \mathrm{~d} \zeta=\frac{(-1)^{m}}{\pi} p !(-N)^{n-p}\left(\begin{array}{c}
n \\
p
\end{array}\right) \int_{0}^{\infty} \frac{\zeta \mathrm{e}^{-N \zeta}}{\varepsilon^{2}+\zeta^{2}} \mathrm{~d} \zeta \\
+\frac{(-1)^{m}}{\pi} p !(-N)^{n-p} \sum_{\ell=1}^{p}\left(\begin{array}{c}
n \\
p-\ell
\end{array}\right) \frac{(-1)^{\ell}}{\ell}+\mathrm{o}(1) .
\end{aligned}
$$

It is clear that when condition (5.24) is satisfied, the contributions coming from the first term of the right-hand side of 5.29 cancel, and we arrive to (5.25).

If condition (5.24) does not hold, then we need to prove that the integral on the righthand side of $(5.29)$ diverges as $\varepsilon \downarrow 0$. To see this, we integrate by parts to get

$$
\int_{0}^{\infty} \frac{\zeta}{\varepsilon^{2}+\zeta^{2}} \mathrm{e}^{-N \zeta} \mathrm{d} \zeta=-\log \varepsilon+\frac{N}{2} \int_{0}^{\infty} \log \left(\varepsilon^{2}+\zeta^{2}\right) \mathrm{e}^{-N \zeta} \mathrm{d} \zeta
$$

and observe that the integral on the right-hand side of $(5.30)$ is $\mathrm{O}(1)$ as $\varepsilon \downarrow 0$.

\section{Moments of characteristic polynomials and their deriva- tives}

We have now collected ingredients required to prove theorem 2.2. After giving the proof below, we then will consider the asymptotics limit $N \rightarrow \infty$ of large matrix size (proposition 6.2 below).

\subsection{The finite $N$ case}

Proof of theorem 2.2. We use proposition 3.2 and proposition 4.4 to write, for $k \in \mathbb{N}$,

$$
\tilde{F}_{N}(h, k)=\lim _{\varepsilon \downarrow 0} \frac{1}{2^{2 h}} \tilde{F}_{N}(0, k) \int_{-\infty}^{\infty} K_{2 h}(\varepsilon, \zeta) \mathrm{e}^{-N|\zeta|}{ }_{1} F_{1}^{(1)}(-k ;-2 k ; 2|\zeta|, \ldots, 2|\zeta|) \mathrm{d} \zeta .
$$

We shall derive an expansion for the hypergeometric function in terms of the coefficients $\mathcal{C}_{N}(p, k)$ defined by 2.5$)$. We begin by using the definition 4.36$)$ to write

$$
{ }_{1} F_{1}^{(1)}(-k ;-2 k ; 2|\zeta|, \ldots, 2|\zeta|)=\sum_{\lambda} \frac{[-k]_{\lambda}}{[-2 k]_{\lambda}} \frac{C_{\lambda}^{(1)}(2|\zeta|, \ldots, 2|\zeta|)}{|\lambda| !} .
$$

From the argument given in section 4.2 , the series in $(6.2)$ is finite. Indeed, the sum runs over only those partitions $\lambda$ with largest part not greater than $k$ (so that the factor $[-2 k]_{\lambda}$ in the denominator is never zero). We use the homogeneity of Jack polynomials, and the normalisation 4.38 to get

$$
\begin{aligned}
{ }_{1} F_{1}^{(1)}(-k ;-2 k ; 2|\zeta|, \ldots, 2|\zeta|) & =\sum_{\lambda} \frac{[-k]_{\lambda}}{[-2 k]_{\lambda}}(2|\zeta|)^{|\lambda|} \frac{C_{\lambda}^{(1)}(1, \ldots, 1)}{|\lambda| !} \\
& =\sum_{\lambda} \frac{[-k]_{\lambda}}{[-2 k]_{\lambda}}(2|\zeta|)^{|\lambda|} \frac{[N]_{\lambda}}{h_{\lambda}^{2}} .
\end{aligned}
$$


We can index the sum in (6.3) by transposes of partitions, rather than the partitions themselves. This gives

$$
\begin{aligned}
{ }_{1} F_{1}^{(1)}(-k ;-2 k ; 2|\zeta|, \ldots, 2|\zeta|) & =\sum_{\lambda} \frac{[-k]_{\lambda^{\mathrm{T}}}}{[-2 k]_{\lambda^{\mathrm{T}}}} \frac{[N]_{\lambda_{\mathrm{T}}}}{h_{\lambda^{\mathrm{T}}}^{2}}(2|\zeta|)^{|\lambda|} \\
& =\sum_{\lambda} \frac{[k]_{\lambda}(-1)^{|\lambda|}[-N]_{\lambda}}{[2 k]_{\lambda} h_{\lambda}^{2}}(2|\zeta|)^{|\lambda|}
\end{aligned}
$$

using (2.4). We will group the terms of (6.4) so that partitions of the same integer $p$ are summed together. Since we transposed the partitions, we know that $\lambda$ can have at most $k$ parts. This manipulation brings us finally to

$$
\begin{aligned}
{ }_{1} F_{1}^{(1)}(-k ;-2 k ; 2|\zeta|, \ldots, 2|\zeta|) & =\sum_{p=0}^{k N}\left(\sum_{\lambda \vdash_{k} p} \frac{[k]_{\lambda}[-N]_{\lambda}}{[2 k]_{\lambda} h_{\lambda}^{2}}\right)(-2|\zeta|)^{p} \\
& =\sum_{p=0}^{k N} \mathcal{C}_{N}(p, k)|\zeta|^{p} .
\end{aligned}
$$

Substituting (6.5) into 6.1) we arrive at

$$
\tilde{F}_{N}(h, k)=\lim _{\varepsilon \downarrow 0} \frac{2}{2^{2 h}} \tilde{F}_{N}(0, k) \sum_{p=0}^{k N} \mathcal{C}_{N}(p, k) \int_{0}^{\infty} K_{2 h}(\varepsilon, \zeta) \mathrm{e}^{-N \zeta} \zeta^{p} \mathrm{~d} \zeta .
$$

We split the sum into two contributions according to $0 \leqslant p \leqslant 2 h$ and $2 h<p \leqslant k N$, and apply proposition 5.3 to the second sum, and apply proposition 5.6 to the first sum. By proposition 3.2 we know a priori that the limit $\varepsilon \downarrow 0$ exists in (6.6), so condition (5.24) must hold with $f_{p}=\mathcal{C}_{N}(p, k)$. This means that in addition to proving (2.9), we have also proved the combinatorial identity

$$
\sum_{p=0}^{2 h}\left(\begin{array}{c}
2 h \\
p
\end{array}\right) \mathcal{C}_{N}(p, k) \frac{p !}{(-N)^{p}}=0
$$

valid for $2 h$ an odd integer with $0<h \leqslant k$.

We remark that starting from equation (6.6) (which does not depend on the parity of $2 h$ ), and using propositions 5.3 and 5.5, we can re-prove Dehaye's result, theorem 2.1. for $2 h$ even and $k$ integer, using our methods.

\subsection{The $N \rightarrow \infty$ limit}

In order to pass to the limit $N \rightarrow \infty$ in 2.9 ) we require an estimate on the size of the coefficients $\mathcal{C}_{N}(p, k)$. This is provided by the following lemma.

Lemma 6.1. Let $N \geqslant 1$ and $p \geqslant 2$. Then

$$
\mathcal{C}_{N}(p, k)=\mathrm{O}_{k}\left(\frac{N^{p}}{p !}\right),
$$

where the implied constant may depend on $k$, but is independent of $N$ and $p$. 
Proof. We use the fact that if $\lambda=\left(\lambda_{1}, \ldots, \lambda_{k}\right)$ is a partition of $p$ into not more than $k$ parts, then $\lambda_{1} \geqslant\left\lfloor\frac{p}{k}\right\rfloor$, where $\lfloor\cdot\rfloor$ is the integer-part function. Then we have,

$$
\begin{aligned}
{[2 k]_{\lambda}=\prod_{i=1}^{k} \prod_{j=1}^{\lambda_{i}}(2 k+j-i) } & \geqslant \prod_{j=1}^{\lambda_{1}}(2 k+j-1) \\
& \geqslant \prod_{j=1}^{\lfloor p / k\rfloor}(2 k+j-1) \\
& =\frac{\Gamma(2 k+\lfloor p / k\rfloor)}{\Gamma(2 k)} .
\end{aligned}
$$

With this inequality, and the trivial estimate

$$
\left|[-N]_{\lambda}\right| \leqslant(N+k)^{p}
$$

we can bound $\mathcal{C}_{N}(p, k)$ as follows:

$$
\begin{aligned}
\left|\mathcal{C}_{N}(p, k)\right|=2^{p}\left|\sum_{\lambda \vdash_{k} p} \frac{[k]_{\lambda}[-N]_{\lambda} \mid}{[2 k]_{\lambda} h_{\lambda}^{2}}\right| & \leqslant \frac{2^{p}(N+k)^{p} \Gamma(2 k)}{\Gamma(2 k+\lfloor p / k\rfloor)} \sum_{\lambda \vdash_{k} p} \frac{[k]_{\lambda}}{h_{\lambda}^{2}} \\
& =\frac{2^{p}(N+k)^{p} \Gamma(2 k)}{\Gamma(2 k+\lfloor p / k\rfloor)} \sum_{\lambda \vdash p} \frac{[k]_{\lambda}}{h_{\lambda}^{2}},
\end{aligned}
$$

where the last equality holds, since $[k]_{\lambda}=0$ if $\lambda$ is a partition with more than $k$ parts. It follows from the hook-content formula (see Theorem 7.21.2 of [49] combined with Proposition 2.2 of [50]) that

$$
\sum_{\lambda \vdash p} \frac{[k]_{\lambda}}{h_{\lambda}^{2}}=\frac{k^{p}}{p !},
$$

so we have proved

$$
\left|\mathcal{C}_{N}(p, k)\right| \leqslant \frac{N^{p}}{p !}\left(1+\frac{k}{N}\right)^{p} \frac{(2 k)^{p} \Gamma(2 k)}{\Gamma(2 k+\lfloor p / k\rfloor)},
$$

which furnishes the required estimate.

Lemma 6.1 is probably far from optimal, but is sufficient to prove the result following.

Proposition 6.2. Let $h=(2 m-1) / 2$ for $m \in \mathbb{N}$ and let $k \in \mathbb{N}$ with $k>h-\frac{1}{2}$. Then

$$
\begin{aligned}
\tilde{F}(h, k)=\frac{2(-1)^{h+1 / 2}}{2^{2 h} \pi} \tilde{F}(0, k)\left\{\sum_{p=1}^{2 h} \sum_{\ell=1}^{p}\left(\begin{array}{c}
2 h \\
p-\ell
\end{array}\right) \frac{(-1)^{\ell+2 h-p}}{\ell} p ! \mathcal{C}(p, k)+\right. \\
\left.\sum_{p=2 h+1}^{\infty}(2 h) !(p-2 h-1) ! \mathcal{C}(p, k)\right\},
\end{aligned}
$$

where

$$
\tilde{F}(0, k)=\prod_{j=1}^{k} \frac{\Gamma(j)}{\Gamma(k+j)} .
$$


Proof. We recall that

$$
\tilde{F}(h, k):=\lim _{N \rightarrow \infty} \frac{1}{N^{k^{2}+2 h}} \tilde{F}_{N}(h, k) .
$$

We can apply the limit term-by-term in the first sum of (2.9), using (2.7) and the fact that

$$
\tilde{F}_{N}(0, k) \sim \tilde{F}(0, k) N^{k^{2}},
$$

as $N \rightarrow \infty$, which was proved in [11].

For the second sum, lemma 6.1 gives us

$$
\frac{(p-2 h-1) !}{N^{p}} \mathcal{C}_{N}(p, k)=\mathrm{O}_{k}\left(\frac{1}{p^{2 h+1}}\right),
$$

so that the second summand of $(2.9)$ is bounded independently of $N$ by a summable function of $p$. Taken together with (2.7), this allows us to apply Tannery's theorem [51, $\S 49]$ to prove

$$
\lim _{N \rightarrow \infty} \sum_{p=2 h+1}^{k N} \frac{(p-2 h-1) !}{N^{p}} \mathcal{C}_{N}(p, k)=\sum_{p=2 h+1}^{\infty}(p-2 h-1) ! \mathcal{C}(p, k),
$$

and hence we get (6.14).

\section{$7 \quad$ Partition sums and a proof of 1.11}

In order to give explicit formulæ for the moment $\tilde{F}(h, k)$ we require closed forms for $\mathcal{C}(p, k)$. We have been able to find these forms for $k=1$ and $k=2$ :

Proposition 7.1. Let $p \in \mathbb{N}$ and $\mathcal{C}(p, k)$ be defined by 2.6). Then,

$$
\mathcal{C}(p, 1)=\frac{2^{p}}{p !(p+1) !},
$$

and

$$
\mathcal{C}(p, 2)=\frac{12(2 p+4) ! 2^{p}}{p !(p+2) !(p+3) !(p+4) !} .
$$

Proof. We recall that

$$
\mathcal{C}(p, k):=2^{p} \sum_{\lambda \vdash_{k} p} \frac{[k]_{\lambda}}{[2 k]_{\lambda} h_{\lambda}^{2}},
$$

where the summation goes over partitions of $p$ into not more than $k$ parts. In the case $k=1$, then only the single partiton $\lambda=(p)$ is admitted. In this case, it is easy to see that $[1]_{\lambda}=p !,[2]_{\lambda}=(p+1)$ ! and $h_{\lambda}=p$ !. This immediately leads to (7.1).

For $k=2$ we require all partitions of $p$ into not more than 2 parts. These partitions are of the form $\lambda=(p-x, x)$, where $0 \leqslant x<(p+1) / 2$ (see figure 2). For a partition of this form, we have

$$
[2]_{\lambda}=(p-x+1) ! x !
$$

and

$$
[4]_{\lambda}=\frac{(x+2) !(p-x+3) !}{12}
$$




\begin{tabular}{|c|c|c|c|c|c|c|c|}
\hline \multicolumn{8}{|c|}{$p-x$ boxes } \\
\hline 0 & 1 & $\cdots$ & $x-1$ & $x$ & $x+1$ & $\cdots$ & $p-x-1$ \\
\hline-1 & 0 & $\cdots$ & $x-2$ & & & & \\
\hline
\end{tabular}

Figure 2: Partitions of $p$ into 2 parts. The contents of each box are the values of $j-i$ appearing in the definition of the Pochhammer symbol.

\begin{tabular}{|c|c|c|c|c|c|c|c|}
\hline$p-x+1$ & $p-x$ & $\cdots$ & $p-2 x+2$ & $p-2 x$ & $\cdots$ & 2 & 1 \\
\hline$x$ & $x-1$ & $\cdots$ & 1 & \multicolumn{3}{|c|}{} \\
\cline { 1 - 3 }
\end{tabular}

Figure 3: The hooks for the partition $\lambda=(p-x, x)$. The hook length $h_{\lambda}$ is the product of the entries in the boxes.

and the hook length $h_{\lambda}$ is given by (see figure 3)

$$
h_{\lambda}=\frac{x !(p-x+1) !}{p-2 x+1},
$$

so that

$$
\mathcal{C}(p, 2)=2^{p} \sum_{0 \leqslant x<(p+1) / 2} \frac{12(p-2 x+1)^{2}}{x !(x+2) !(p-x+3) !(p-x+1) !} .
$$

We observe that the summand in (7.6) is invariant under the reflection $x \mapsto p+1-x$, so that the half-range sum can be replaced by one half times the sum from 0 to $p+1$, giving

$$
\mathcal{C}(p, 2)=2^{p} 6 \sum_{x=0}^{p+1} \frac{(p-2 x+1)^{2}}{x !(x+2) !(p-x+3) !(p-x+1) !} .
$$

In appendix B.2 the sum in (7.7) is evaluated, whereupon $(7.2)$ follows.

Based on the results of proposition 7.1, it is tempting to conjecture that $\mathcal{C}(p, k)$ will be $2^{p}$ times a ratio of products of factorials for all $k \in \mathbb{N}$. However a computer-based investigation of $\mathcal{C}(p, 3)$ has shown that this structure appears to break down when $k=3$.

When $h=1 / 2$ and $k=1$, substituting (7.1) into equation (6.14) gives

$$
\begin{aligned}
\tilde{F}\left(\frac{1}{2}, 1\right) & =\frac{1}{\pi}\left(1-\sum_{p=2}^{\infty} \frac{2^{p}(p-2) !}{p !(p+1) !}\right) \\
& =\frac{\mathrm{e}^{2}-5}{4 \pi}
\end{aligned}
$$

as conjectured.

Using (7.2) for the case $k=2$ in 6.14 , we can evaluat $\AA^{3}$

$$
\begin{aligned}
\tilde{F}\left(\frac{1}{2}, 2\right) & =\frac{1}{180 \pi}\left(15-7_{3} F_{3}\left(1,1, \frac{9}{2} ; 3,6,7 ; 8\right)\right) \\
& \approx 0.00815 \ldots
\end{aligned}
$$

\footnotetext{
${ }^{3}$ We have used the algebraic manipulation package Maple to derive closed forms for the sums in terms of hypergeometric functions.
} 
and

$$
\begin{aligned}
\tilde{F}\left(\frac{3}{2}, 2\right) & =\frac{1}{10080 \pi}\left(33_{3} F_{3}\left(1,1, \frac{13}{2} ; 5,8,9 ; 8\right)-28\right) \\
& \approx 0.000354 \ldots
\end{aligned}
$$

These last two values do not appear to have been derived or conjectured before.

\section{Acknowledgements}

I am grateful for a number of interesting conversations regarding this work with Gregory Berkolaiko, Chris Hughes, Paul-Olivier Dehaye, Martin Hallnäs and Ian Thompson. Jon Keating provided a number of crucial suggestions. I am grateful to Peter Forrester for bringing reference [48] to my attention.

\section{A An elementary evaluation of $\tilde{F}\left(\frac{1}{2}, 1\right)$}

In this appendix we give a completely elementary derivation of (1.11) based on classical properties of Laguerre polynomials (see, for example, [35]).

We first recall the alternative expression (5.3) for $K_{1}(\varepsilon, \zeta)$ :

$$
K_{1}(\varepsilon, \zeta)=\frac{1}{\pi} \frac{\partial}{\partial \zeta}\left(\frac{\zeta}{\varepsilon^{2}+\zeta^{2}}\right) .
$$

Equipped with this, we proceed from the integral representation of proposition 3.2 and equation 4.28,

$$
\tilde{F}_{N}\left(\frac{1}{2}, 1\right)=\lim _{\varepsilon \downarrow 0} \frac{1}{2} \int_{-\infty}^{\infty} K_{1}(\varepsilon, \zeta) \mathrm{e}^{-N|\zeta|} L_{N}^{(1)}(-2|\zeta|) \mathrm{d} \zeta .
$$

Substituting (A.1) and integrating-by-parts, we get

$$
\begin{aligned}
\frac{1}{\pi} \int_{0}^{\infty} K_{1}(\varepsilon, \zeta) \mathrm{e}^{-N \zeta} L_{N}^{(1)}(-2 \zeta) \mathrm{d} \zeta & =-\frac{1}{\pi} \int_{0}^{\infty} \frac{\zeta}{\varepsilon^{2}+\zeta^{2}} \frac{\partial}{\partial \zeta}\left(\mathrm{e}^{-N \zeta} L_{N}^{(1)}(-2 \zeta)\right) \mathrm{d} \zeta \\
& =\frac{1}{\pi} \int_{0}^{\infty} \frac{\zeta}{\varepsilon^{2}+\zeta^{2}} \mathrm{e}^{-N \zeta}\left(N L_{N}^{(1)}(-2 \zeta)-2 L_{N-1}^{(2)}(-2 \zeta)\right) \mathrm{d} \zeta
\end{aligned}
$$

where we have used the fact that

$$
\frac{\mathrm{d}}{\mathrm{d} t} L_{N}^{(\alpha)}(t)=-L_{N-1}^{(\alpha+1)}(t) .
$$

By a standard recurrence for Laguerre polynomials,

$$
N L_{N}^{(1)}(-2 \zeta)-2 L_{N-1}^{(2)}(-2 \zeta)=2 \zeta L_{N-1}^{(3)}(-2 \zeta),
$$

so that we have

$$
\tilde{F}_{N}\left(\frac{1}{2}, 1\right)=\frac{2}{\pi} \lim _{\varepsilon \downarrow 0} \int_{0}^{\infty} \frac{\zeta^{2}}{\varepsilon^{2}+\zeta^{2}} \mathrm{e}^{-N \zeta} L_{N-1}^{(3)}(-2 \zeta) \mathrm{d} \zeta
$$


Since

$$
0 \leqslant \frac{\zeta^{2}}{\varepsilon^{2}+\zeta^{2}} \leqslant 1
$$

we can apply the dominated convergence theorem to pass the limit under the integral, to get

$$
\tilde{F}_{N}\left(\frac{1}{2}, 1\right)=\frac{2}{\pi} \int_{0}^{\infty} \mathrm{e}^{-N \zeta} L_{N-1}^{(3)}(-2 \zeta) \mathrm{d} \zeta
$$

At this point, we insert (4.18), the explicit representation of $L_{N}^{(\alpha)}$, getting that

$$
\begin{aligned}
\int_{0}^{\infty} \mathrm{e}^{-N \zeta} L_{N-1}^{(3)}(-2 \zeta) \mathrm{d} \zeta & =\sum_{n=0}^{N-1}\left(\begin{array}{c}
N+2 \\
n+3
\end{array}\right) \frac{2^{n}}{n !} \int_{0}^{\infty} \mathrm{e}^{-N \zeta} \zeta^{n} \mathrm{~d} \zeta \\
& =\sum_{n=0}^{N-1}\left(\begin{array}{c}
N+2 \\
n+3
\end{array}\right) \frac{2^{n}}{N^{n+1}} .
\end{aligned}
$$

So,

$$
\tilde{F}_{N}\left(\frac{1}{2}, 1\right)=\frac{2}{\pi} \sum_{n=0}^{N-1}\left(\begin{array}{c}
N+2 \\
n+3
\end{array}\right) \frac{2^{n}}{N^{n+1}}
$$

and

$$
\tilde{F}\left(\frac{1}{2}, 1\right)=\lim _{N \rightarrow \infty} \frac{1}{N^{2}} \tilde{F}_{N}\left(\frac{1}{2}, 1\right)=\lim _{N \rightarrow \infty} \sum_{n=0}^{N-1}\left(\begin{array}{c}
N+2 \\
n+3
\end{array}\right) \frac{2^{n}}{N^{n+3}} .
$$

To perform the last sum, we use the estimate $\left(\begin{array}{c}N+2 \\ n+3\end{array}\right) \leqslant \frac{(N+2)^{n+3}}{(n+3) !}$ to get

$$
\begin{aligned}
\left(\begin{array}{c}
N+2 \\
n+3
\end{array}\right) \frac{2^{n}}{N^{n+3}} & \leqslant \frac{2^{n}}{(n+3) !}\left(\frac{N+2}{N}\right)^{n+3} \\
& \leqslant \frac{2^{n}}{n !} \mathrm{e}^{2},
\end{aligned}
$$

which is summable. This allows us to use Tannery's theorem [51, §49]. Since

$$
\lim _{N \rightarrow \infty} \frac{1}{N^{n+3}}\left(\begin{array}{c}
N+2 \\
n+3
\end{array}\right)=\frac{1}{(n+3) !}
$$

by Stirling's approximation, we get

$$
\begin{aligned}
\tilde{F}\left(\frac{1}{2}, 1\right) & =\frac{2}{\pi} \sum_{n=0}^{\infty} \frac{2^{n}}{(n+3) !} \\
& =\frac{\mathrm{e}^{2}-5}{4 \pi} .
\end{aligned}
$$

\section{B Some sums}

In this appendix we give evaluations of some finite sums that appear in the main text. 


\section{B.1 A sum from section 5}

In this appendix we evaluate the sum

$$
\sum_{\ell=0}^{n}(-1)^{\ell}\left(\begin{array}{c}
p \\
n-\ell
\end{array}\right)\left(\begin{array}{c}
p-n+\ell-1 \\
\ell
\end{array}\right)
$$

for $p \geqslant n+1$.

By elementary manipulation of binomial coefficients,

$$
\begin{aligned}
\left(\begin{array}{c}
p \\
n-\ell
\end{array}\right)\left(\begin{array}{c}
p-n+\ell+1 \\
\ell
\end{array}\right) & =\frac{p !(p-n+\ell-1) !}{(n-\ell) !(p-n+\ell) ! \ell !(p-n-1) !} \\
& =\frac{p}{p-n+\ell}\left(\begin{array}{c}
p-1 \\
n
\end{array}\right)\left(\begin{array}{c}
n \\
\ell
\end{array}\right) .
\end{aligned}
$$

We also use the fact that

$$
\int_{0}^{1} x^{p-1-n+\ell} \mathrm{d} x=\frac{1}{p-n+\ell}
$$

together with $(\mathrm{B} .2)$ to get

$$
\begin{aligned}
\sum_{\ell=0}^{n}(-1)^{\ell}\left(\begin{array}{c}
p \\
n-\ell
\end{array}\right)\left(\begin{array}{c}
p-n+\ell-1 \\
\ell
\end{array}\right) & =p\left(\begin{array}{c}
p-1 \\
n
\end{array}\right) \sum_{\ell=0}^{n}(-1)^{\ell}\left(\begin{array}{c}
n \\
\ell
\end{array}\right) \int_{0}^{1} x^{p-1-n+\ell} \mathrm{d} x \\
& =p\left(\begin{array}{c}
p-1 \\
n
\end{array}\right) \int_{0}^{1} x^{p-n-1}(1-x)^{n} \mathrm{~d} x \\
& =p\left(\begin{array}{c}
p-1 \\
n
\end{array}\right) \frac{(p-n-1) ! n !}{p !}=1
\end{aligned}
$$

We have used

$$
\int_{0}^{1} x^{a}(1-x)^{b} \mathrm{~d} x=\frac{a ! b !}{(a+b+1) !}, \quad \text { for } a, b \in \mathbb{N}:
$$

a version of Euler's integral.

\section{B.2 A sum from section 7}

In this appendix we evaluate the sum

$$
\sum_{x=0}^{p+1} \frac{(p-2 x+1)^{2}}{x !(x+2) !(p-x+3) !(p-x+1) !} .
$$

Let us observe that the numerator in $(\mathrm{B} .6)$ can be written as

$$
(p-2 x+1)^{2}=(p-x+1)(p-x+3)+x(x+2)-(p-x+1)(x+2)-x(p-x+3) .
$$


Therefore,

$$
\begin{aligned}
& \sum_{x=0}^{p+1} \frac{(p-2 x+1)^{2}}{x !(x+2) !(p-x+3) !(p-x+1) !} \\
& =\sum_{x=0}^{p} \frac{1}{x !(x+2) !(p-x+2) !(p-x) !}+\sum_{x=1}^{p+1} \frac{1}{(x-1) !(x+1) !(p-x+3) !(p-x+1) !} \\
& \quad-\sum_{x=0}^{p} \frac{1}{x !(x+1) !(p-x+3) !(p-x) !}-\sum_{x=1}^{p+1} \frac{1}{(x-1) !(x+2) !(p-x+2) !(p-x+1) !} \\
& =\frac{1}{(p+2) !^{2}}\left(\sum_{x=0}^{p}\left(\begin{array}{c}
p+2 \\
x+2
\end{array}\right)\left(\begin{array}{c}
p+2 \\
x
\end{array}\right)+\sum_{x=1}^{p+1}\left(\begin{array}{c}
p+2 \\
x-1
\end{array}\right)\left(\begin{array}{c}
p+2 \\
x+1
\end{array}\right)\right) \\
& \quad-\frac{1}{(p+1) !(p+3) !}\left(\begin{array}{c}
p \\
x=0
\end{array}\left(\begin{array}{c}
p+1 \\
x+1
\end{array}\right)\left(\begin{array}{c}
p+3 \\
x
\end{array}\right)+\sum_{x=1}^{p+1}\left(\begin{array}{c}
p+1 \\
x-1
\end{array}\right)\left(\begin{array}{c}
p+3 \\
x+2
\end{array}\right)\right) \\
& \quad-\frac{1}{(p+2) !^{2}} \sum_{x=0}^{p}\left(\begin{array}{c}
p+2 \\
x
\end{array}\right)\left(\begin{array}{c}
p+2 \\
p-x
\end{array}\right) \\
& \quad \frac{1}{(p+1) !(p+3) !}\left(\sum_{x=0}^{p}\left(\begin{array}{c}
p+3 \\
x
\end{array}\right)\left(\begin{array}{c}
p+1 \\
p-x
\end{array}\right)+\sum_{x=0}^{p}\left(\begin{array}{c}
p+1 \\
x
\end{array}\right)\left(\begin{array}{c}
p+3 \\
p-x
\end{array}\right)\right) .
\end{aligned}
$$

We then use the Vandermonde identity,

$$
\sum_{x=0}^{p}\left(\begin{array}{c}
w \\
x
\end{array}\right)\left(\begin{array}{c}
v \\
p-x
\end{array}\right)=\left(\begin{array}{c}
w+v \\
p
\end{array}\right),
$$

to get

$$
\begin{aligned}
\sum_{x=0}^{p+1} \frac{(p-2 x+1)^{2}}{x !(x+2) !(p-x+3) !(p-x+1) !} & =\frac{2}{(p+2) !^{2}}\left(\begin{array}{c}
2 p+4 \\
p
\end{array}\right) \\
& =\frac{-\frac{2}{(p+1) !(p+3) !}\left(\begin{array}{c}
2 p+4 \\
p
\end{array}\right)}{(p+1) !(p+2) !}\left(\frac{1}{p+2}-\frac{1}{p+3}\right)\left(\begin{array}{c}
2 p+4 \\
p
\end{array}\right) \\
& =\frac{2}{(p+2) !(p+3) !}\left(\begin{array}{c}
2 p+4 \\
p
\end{array}\right) .
\end{aligned}
$$

\section{References}

[1] H. Montgomery "The pair correlation of zeros of the zeta function," in Analytic Number Theory vol. 24 of Proc. Symp. Pure Math. pp. 181-193 Amer. Math. Soc. 1973.

[2] D. A. Hejhal (1994) "On the triple correlation of zeros of the zeta function," Int. Math. Res. Not. 7, pp. 293-302. 
[3] Z. Rudnick and P. Sarnak (1994) "The $n$-level correlations of zeros of the zeta function," C. R. Acad. Sci. Paris Sér. I Math. 319, pp. 1027-1032.

[4] Z. Rudnick and P. Sarnak (1996) "Zeros of principal $L$-functions and random matrix theory," Duke Math. J. 81, pp. 269-322.

[5] E. B. Bogomolny and J. P. Keating (1995) "Random matrix theory and the Riemann zeros I: three- and four-point correlations," Nonlinearity 8, pp. 1115-1131.

[6] E. B. Bogomolny and J. P. Keating (1996) "Random matrix theory and the Riemann zeros II: $n$-point correlations," Nonlinearity 9, pp. 911-935.

[7] E. B. Bogomolny and J. P. Keating (1996) "Gutzwiller's trace formula and spectral statistics: Beyond the diagonal approximation," Phys. Rev. Lett. 77, pp. 1472-1475.

[8] A. M. Odlyzko (1987) "On the distribution of spacings between zeros of the zeta function," Math. Comp. 48, pp. 273-308.

[9] A. M. Odlyzko "The $10^{22}$-nd zero of the Riemann zeta function," in Dynamical, spectral, and arithmetic zeta functions (San Antonio, TX, 1999) vol. 290 of Contemp. Math. pp. 139-144 Providence, RI: Amer. Math. Soc. 2001.

[10] G. A. Hiary and A. M. Odlyzko "The zeta function on the critical line: Numerical evidence for moments and random matrix theory models." Math. Comp. to appear.

[11] J. P. Keating and N. C. Snaith (2000) "Random matrix theory and $\zeta(1 / 2+\mathrm{i})$," Commun. Math. Phys. 214, pp. 57-89.

[12] J. P. Keating and N. C. Snaith (2000) "Random matrix theory and $L$-functions at $s=1 / 2$," Commun. Math. Phys. 214, pp. 91-110.

[13] C. P. Hughes, J. P. Keating, and N. O'Connell (2000) "Random matrix theory and the derivative of the Riemann zeta function," Proc. R. Soc. Lond. A 456, pp. 2611-2627.

[14] C. P. Hughes, J. P. Keating, and N. O'Connell (2001) "On the characteristic polynomial of a random unitary matrix," Commun. Math. Phys. 220, pp. 429-451.

[15] J. B. Conrey, D. W. Farmer, J. P. Keating, M. O. Rubinstein, and N. C. Snaith (2003) "Autocorrelation of random matrix polynomials," Commun. Math. Phys. 237, pp. 365-395.

[16] J. B. Conrey, D. W. Farmer, J. P. Keating, M. O. Rubinstein, and N. C. Snaith (2005) "Integral moments of L-functions," Proc. Lond. Math. Soc. (3) 91, pp. 33-104.

[17] J. B. Conrey, D. W. Farmer, J. P. Keating, M. O. Rubinstein, and N. C. Snaith (2008) "Lower order terms in the full moment conjecture for the Riemann zeta function," $J$. Number Theory 128, pp. 1516-1554.

[18] J. B. Conrey "L-functions and random matrices," in Mathematics Unlimited 2001 and Beyond (B. Enquist and W. Schmidt, eds.) pp. 331-352 2001.

[19] J. P. Keating and N. C. Snaith (2003) "Random matrices and L-functions," J. Phys. A 36, pp. 2859-2881. 
[20] J. P. Keating "Random matrices and number theory," in Applications of Random Matrices in Physics (E. Brézin, V. Kazakov, D. Serban, P. Wiegmann, and A. Zabrodin, eds.) vol. 221 of NATO Science pp. 1-32 2006.

[21] N. C. Snaith (2010) "Riemann zeros and random matrix theory," Milan J. Math. 78, pp. 135-152.

[22] R. R. Hall (2002) "A Wirtinger type inequality and the spacing of the zeros of the Riemann zeta-function," J. Number Theory 93, pp. 235-245.

[23] J. B. Conrey and A. Ghosh (1984) "On mean values of the zeta-function," Mathematika 31, pp. 159-161.

[24] C. P. Hughes On the Characteristic Polynomial of a Random Unitary Matrix and the Riemann Zeta Function. Ph.D. thesis, University of Bristol 2001.

[25] P.-O. Dehaye (2008) "Joint moments of derivatives of characteristic polynomials," Alg. 85 Num. Th. 2, pp. 31-68.

[26] P.-O. Dehaye "A note on moments of derivatives of characteristic polynomials," in Formal Power Series and Algebraic Combinatorics vol. 12 of DMTCS Proc. pp. 6456562010.

[27] A. E. Ingham (1926) "Mean-value theorems in the theory of the Riemann zeta function," Proc. Lond. Math. Soc. 27, pp. 273-300.

[28] J. B. Conrey (1988) "The fourth moment of derivatives of the Riemann zeta-function," Quart. J. Math. Oxford 39, pp. 21-36.

[29] J. B. Conrey and A. Ghosh (1989) "On mean values of the zeta-function II," Acta Arithmetica 52, pp. 367-371.

[30] J. B. Conrey and A. Ghosh (1985) "A mean value theorem for the Riemann zetafunction at its relative extrema on the critical line," J. Lond. Math. Soc. 32, pp. 193202.

[31] A. C. Aitken Determinants and Matrices. Oliver and Boyd 1942.

[32] I. S. Gradshteyn and I. M. Ryzhik Table of integrals, series, and products. Elsevier/Academic Press, Amsterdam 7th ed. 2007.

[33] M. Abramowitz and I. A. Stegun Handbook of Mathematical Functions. Dover 1970.

[34] E. Brézin and S. Hikami (2000) "Characteristic polynomials of random matrices," Comm. Math. Phys. 214, pp. 111-135.

[35] G. Szegő Orthogonal polynomials. American Mathematical Society Colloquium Publications, Vol. 23. Revised ed Providence, R.I.: American Mathematical Society 1959.

[36] W. N. Bailey Generalized Hypergeometric Series. Stechert-Hafner 1964.

[37] A. Korányi "Hua-type integrals, hypergeometric functions and symmetric polynomials," in International Symposium in Memory of Hua Loo Keng, Vol II pp. 169-180 Springer, Berlin 1991. 
[38] J. Kaneko (1993) "Selberg integrals and hypergeometric functions associated with Jack polynomials," SIAM J. Math. Anal. 24, pp. 1086-1110.

[39] Z. Yan (1992) "A class of generalized hypergeometric functions in several variables," Canad. J. Math. 44, pp. 1317-1338.

[40] A. G. Constantine (1963) "Some noncentral distribution problems in multivariate analysis," Ann. Math. Statist. 34, pp. 1270-1285.

[41] P. R. Krishnaiah and T. C. Chang (1971) "On the exact distribution of the smallest root of the Wishart matrix using zonal polynomials," Ann. Math. Statist. pp. 293-295.

[42] I. Dumitriu Eigenvalue Statistics for the Beta-Ensembles. Ph.D. thesis, Masachusetts Institute of Technology 2003.

[43] I. Dumitriu and P. Koev (2008) "Distributions of the extreme eigenvalues of betaJacobi random matrices," SIAM J. Matrix Anal. Appl. 30, pp. 1-6.

[44] P. J. Forrester and N. S. Witte (2005) "Discrete Painlevé equations for a class of $P_{\mathrm{VI}}$ $\tau$-functions given as $\mathrm{U}(N)$ averages," Nonlinearity 18, pp. 2061-2088.

[45] P. J. Forrester and J. P. Keating (2004) "Singularity dominated strong fluctuations for some random matrix averages," Commun. Math. Phys. 250, pp. 119-131.

[46] R. P. Stanley (1989) "Some combinatorial properties of Jack symmetric functions," Adv. Math. 77, pp. 76-115.

[47] P. J. Forrester Log-gasses and Random Matrices. Princeton University Press 2010.

[48] A. Borodin and G. Olshanski (2005) " $Z$-measures on partitions and their scaling limits," Electronic J. Combinatorics 26, pp. 795-834.

[49] R. P. Stanley Enumerative Combinatorics vol. 2. Cambridge University Press 2001.

[50] P. J. Hanlon, R. Stanley, and J. R. Stembridge (1992) "Some combinatorial aspects of normally distributed random matrices," Contemp. Math. 158, pp. 151-174.

[51] T. J. I'A. Bromwich An Introduction to the Theory of Infinite Series. MacMillan 2nd ed. 1926. 